\title{
WHAT MAKES GEEKS TICK? A STUDY OF STACK OVERFLOW CAREERS
}

\author{
Lei Xu \\ Toulouse School of Economics \\ Tingting Nian \\ University of California, Irvine \\ Luís Cabral \\ New York University and CEPR
}

February 16, 2018

\begin{abstract}
Many online platforms rely on users to voluntarily provide content. What motivates users to contribute content for free however is not well understood. In this paper, we use a revealed preference approach to show that career concerns play an important role in user contributions to Stack Overflow, the largest online Q\&A community. We investigate how activities that can enhance a user's reputation vary before and after the user finds a new job. We contrast this with activities that do not improve a user's reputation. After finding a new job, users contribute $23.7 \%$ less in reputation-generating activity. By contrast, they reduce their non-reputation-generating activity by only $7.4 \%$ after finding a new job. These findings suggest that users contribute to Stack Overflow in part because they perceive this as a way to improve future employment prospects. We provide direct evidence against alternative explanations such as integer constraints, skills mismatch, and dynamic selection effects.
\end{abstract}

JEL Classification Numbers: H41, D82, D83, J24, J22, M51, L86

\section{Introduction}

A fascinating and economically important consequence of the rise of the Internet is the growing prevalence of private contributions to collective projects such as Wikipedia, bulletin boards, or open source software. As Lerner and Tirole (2002) put it, to an economist the behavior of individual contributors appears somewhat puzzling: is it a case of altruism, or are there ulterior motives behind private contributions to a public good?

Our paper addresses this research question using data from Stack Overflow (SO), the largest online Q\&A platform for programming-related matters. We consider a hypothesis put forward by Lerner and Tirole (2002), namely that contributions are motivated by career concerns: the desire to signal one's ability so as to obtain better employment. 1

\footnotetext{
${ }^{1}$ In Holmström's (1982/99) classic theory of career concerns, performance in the current job serves as a signal of one's ability to future employers. A job seeker makes effort to improve current performance in order to signal a higher ability, thus earning a higher salary from the new job. In this paper, "career incentive" and "career concern" are used interchangeably to denote any career-related incentives, such as salary increased, more job offers, etc. In Section 6 we provide an in-depth discussion of the information being signaled through online activity.
} 
Affiliated with SO, the Stack Overflow Careers (SOC) site hosts job listings and contributors' CVs so as to match employers and employees ${ }^{2}$ The data from SO and SOC allows us to link online activity to real-world individuals. We construct complete histories of each individual's online trajectory, including contributions to SO, individual characteristics and employment history. We test the career-concerns hypothesis by identifying shifts in behavior following employment changes. We find that before changing to a new job, a contributor provides more and better Answers. However, immediately following the job change, we observe a significant drop in both the quantity and quality of Answers activity. This is consistent with a career-concerns theory of contributions to SO: before a job change, a contributor's reputation has an effect on employment prospects, whereas after the job change has taken place, the reputation-employment link disappears or at least is significantly weakened.

The causal link inherent to the career-concerns hypothesis cannot be established based on this piece of evidence alone. A job seeker's behavior can be explained by multiple confounding factors. Most importantly, users starting new jobs may have busier work schedules which prevents them from contributing to SO. Accordingly, we adopt a modified version of the difference-in-differences (DD) approach: we compare each individual's behavior across different types of activities before and after a job change.

In order to show the rationale behind this approach, we first build a theoretical model of user contributions. We assume that agents derive utility from different activities and are subject to an aggregate time constraint. Specifically, there are three different activities: online contributions that improve an agent's reputation; online contributions that have no effect on an agent's reputation; and work (revenue generating) activities. Finally, we assume that the probability of finding a new job (a better revenue-generating activity) increases an agent's reputation.

The model's equilibrium implies that, upon obtaining a new job, the relative time spent on reputationincreasing online activities (relative to not-reputation-increasing online activity) decreases. This theoretical result forms the basis of our empirical identification strategy.

In particular, the DD approach compares reputation-increasing to not-reputation-increasing activities from the same sample of job changers before and after a job switch $3^{3}$ We conclude that contribution levels decrease by $23.7 \%$ right after a job change, of which $12.4-16.3 \%$ are due to (the removal of) career concerns. Apart from examining both short and long-term activity changes over time, we also consider the heterogeneous responses to job changes for users with different characteristics, such as levels of education, types of degrees, work experience, and existing online reputation. All of the results are consistent with the

\footnotetext{
${ }^{2}$ Job candidates include employment history as well as summary statistics about their contributions to SO.

${ }^{3}$ The vast majority of users have a low level of activity. Our research focuses on the select sample of active contributors. See Section 4 for more details.
} 
career-concerns hypothesis. As with any other DD specification, the validity of our identification hinges on the parallel-trend assumption. We address several major alternative explanations that can potentially invalidate this assumption, including integer constraints, skills mismatch, and dynamic selection effects $\sqrt[4]{4}$ We test the external validity of our results by using a different dataset, one comprised of information from LinkedIn pages rather than $\mathrm{SOC}^{5}$

Our results contribute to understanding the motivations behind online voluntary contributions. We show clear evidence of a widely-held hypothesis: career concerns matter. To the best of our knowledge, this is the first paper that empirically identifies and estimates the causal relation between changes in career status and voluntary contributions to online public goods as an indirect measure of career concerns. We believe our methodology is helpful in other contexts; and we believe our empirical results are important, considering the increasing use of online activity in hiring decisions.

Our results also have important policy implications for platform companies. The prevalence of online platforms has attracted many firms to adopt a platform-based business model. Many tried but failed to launch a successful platform, mostly due to insufficient user participation from one or multiple sides. Due to network effects, a user will not participate without the participation of others. A thorough understanding of the motivations behind user participation is therefore crucial for the success of a platform, especially a platform that relies on voluntary contributions of user-generated content (i.e. a crowdsourcing-based platform). Our results imply that career concerns can be a way through which platforms encourage active user engagement. In Section 8 we develop this and other managerial implications in greater detail.

Related literature. Our research touches on two major streams of literature: career concerns and private contributions to online public goods.

A large body of literature on the theory of career concerns starts with Holmström (1982/99) and Gibbons and Murphy (1992). In contrast to classic models of career concerns, our model abstracts away from the Principal-Agent problem and instead focuses on the time allocation decisions of a job seeker, which provides intuition and guidance to our empirical strategy. The empirical work that estimates the causal effect of career incentives on individuals' behavior is relatively scarce: Chevalier and Ellison (1999) examine the role of career concerns in investment strategies adopted by mutual fund managers; Miklós-Thal and Ullrich

\footnotetext{
${ }^{4}$ In brief, by integer constraints we mean the situation whereby a busier work schedule does not leave enough free time to write Answers, since these take more time than Edits; skills mismatch corresponds to the situation where a new job requires a different set of skills, and a contributor is unable to write Answers related to the new required skills; and the dynamic selection effect (known as Ashenfelter's Dip in labor economics literature) refers to the situation where the sample of job changers only consists of those who happen to contribute many Answers, thus the drop in Answers activity is nothing more than a reversion-to-the-mean effect.

${ }^{5}$ In addition to providing independent evidence of career-concerns effects, our LinkedIn-based alternative dataset addresses potential selection concerns from using SOC pages, given that inclusion in SOC is limited to a set of invited contributors who choose to link their $\mathrm{CV}$ to their SO activity page.
} 
(2015) estimates career concerns of professional soccer players. Our identification strategy is similar to these papers as we also take advantage of the behavioral changes when an agent is facing job terminations.

The literature on online public goods has focused on various types of motivations behind voluntary contributions. For example, Zhang and Zhu (2011) and Algan, Benkler, and Morell (2013) study social effects on Wikipedia, whereas Luca and Zervas (2015) investigate economic incentives for fraudulent reviews on Yelp. In many ways, the type of voluntary contribution that is most similar to Stack Overflow is Open Source Software (OSS). Lakhani and von Hippel (2003) study learning motivation in the context of the Apache field support system; Athey and Ellison (2014) argues that programmers are motivated by reciprocal altruism; Goes, Guo, and Lin (2016) study how setting goals affect users' contribution efforts on a Q\&A website. Conceptually, von Krogh et al. (2012) distinguish three types of motivation: intrinsic (e.g., altruism, ideology, fun, kinship); internalized extrinsic (e.g., reputation, learning, reciprocity, own-use); and extrinsic (e.g., career concerns, pay). 6

There has also been a large literature arguing the career benefits of OSS development. At a conceptual level, Lerner and Tirole (2002, 2005) discussed the hypothesis that OSS contributions are motivated by career concerns. Our paper is a direct empirical test of their hypothesis. Moreover, they conjecture that the incentive for OSS participation is stronger if (i) performance becomes more visible to the relevant audience, (ii) effort has a stronger impact on performance, and (iii) performance becomes more informative about talent. Spiegel (2009) formalizes this conjecture; he shows that it does not always hold, and that multiple equilibria can exist where both positive effort and no effort are plausible. Blatter and Niedermayer (2008) focus on one specific mechanism through which OSS contribution helps career prospects, namely, increased bargaining power during wage renegotiation.

At an empirical level, Roberts, Hann, and Slaughter (2006) and Hann, Roberts, and Slaughter (2013) find evidence of financial returns from participation in OSS projects. Huang and Zhang (2016) demonstrate that active OSS contribution is associated with higher likelihood of job changes. In our theoretical model, the agent takes as given (or believes) that greater online contribution improves job prospects; and chooses the utility-maximizing effort level. Our research focuses on the latter part of the previous sequence, namely the agent's effort choices under the career-benefit belief. Related to our finding, Bitzer and Geishecker (2010) show that the propensity to work on OSS projects is higher among university dropouts, a pattern which they interpret as evidence of career-oriented motivations.

The behavioral economics literature has also studied extensively prosocial behaviors resulting from both

\footnotetext{
${ }^{6}$ There are many other papers that investigate various motivations behind contribution to OSS projects. See von Krogh and von Hippel (2006), von Krogh et al. (2012), and Osterloh and Rota (2007) for detailed surveys of this literature.
} 


\section{RegEx for distance in metric system}

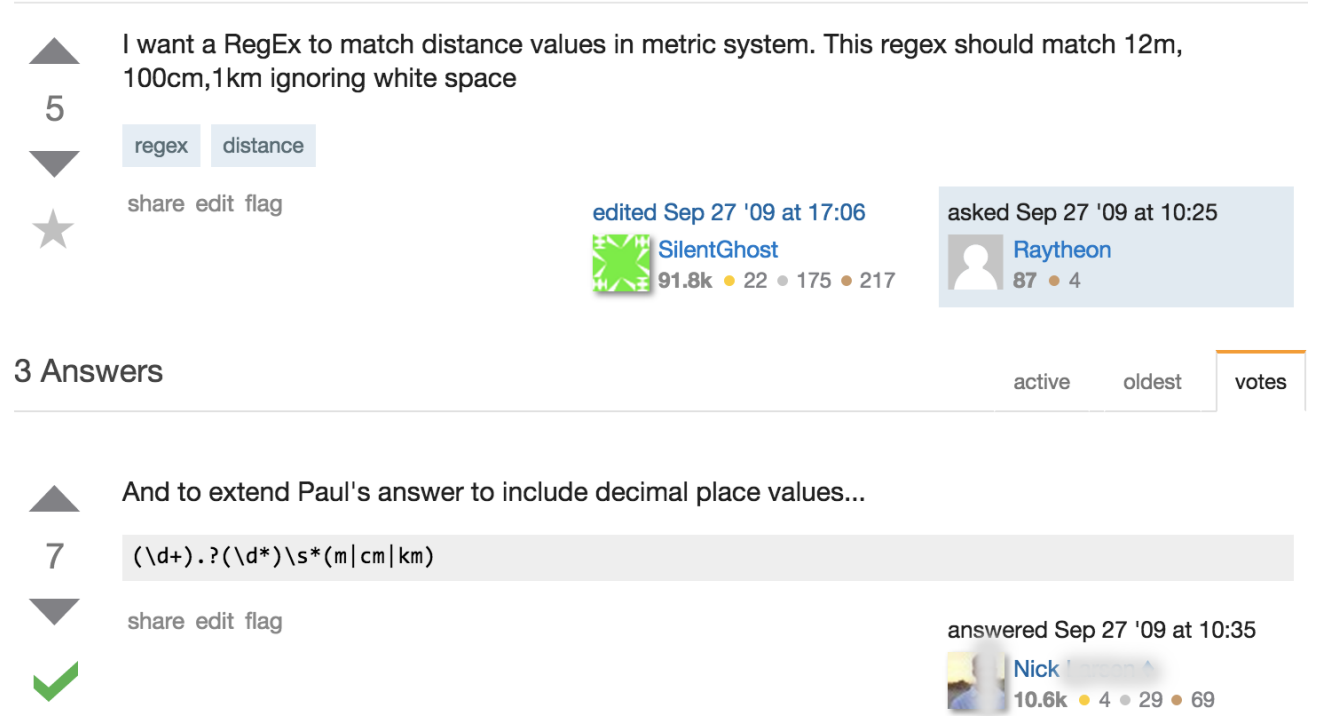

FIGURE 1: A Question with its Answers on Stack Overflow

intrinsic and extrinsic motivations. In this context, one important debate (starting from Titmuss and others (1970) ) relates to the crowd-out effects of extrinsic over intrinsic motivations. The evidence is mixed; see Benabou and Tirole (2003, 2006), Ariely, Bracha, and Meier (2009), and Ashraf, Bandiera, and Lee (2014).

\section{Background}

Stack Overflow (SO) is the largest online Q\&A site where programmers ask and answer programmingrelated questions (Figure 1). It provides for Wikipedia-style editing (Figure 2); and it includes a system of votes, badges and user reputation that ensures high-quality, peer-reviewed answers. SO is widely used by programmers $\sqrt{7}$

Measures of user activity. There are four major activities by users on SO:

Questions Any registered user can ask a Question. A Question can be voted up or down. A hard but important Question is usually voted up to get attention from more contributors. A duplicate or unclear Question is usually voted down 9

\footnotetext{
${ }^{7}$ Founded in 2008 , it currently comprises 4.8 million users. Some summary statistics regarding the site's activity: 7.7 million visits/day; 7.9 thousand questions/day; 10 million cumulative questions, 17 million cumulative Answers.

${ }^{8}$ Stack Overflow is the earliest website of Stack Exchange, which has a network of 150+ Q\&A communities that covers both programming and non-programming related sites. Our research focuses on Stack Overflow, which is the most active community, and it holds $67 \%$ of Q\&As and 52\% of daily visits of the whole Stack Exchange Network as of 2016. Please check http://stackexchange.com/sites for more details.

${ }^{9}$ Our analysis focuses mostly on Answers activity instead of Questions activity for two main reasons: first, ability-signaling
} 


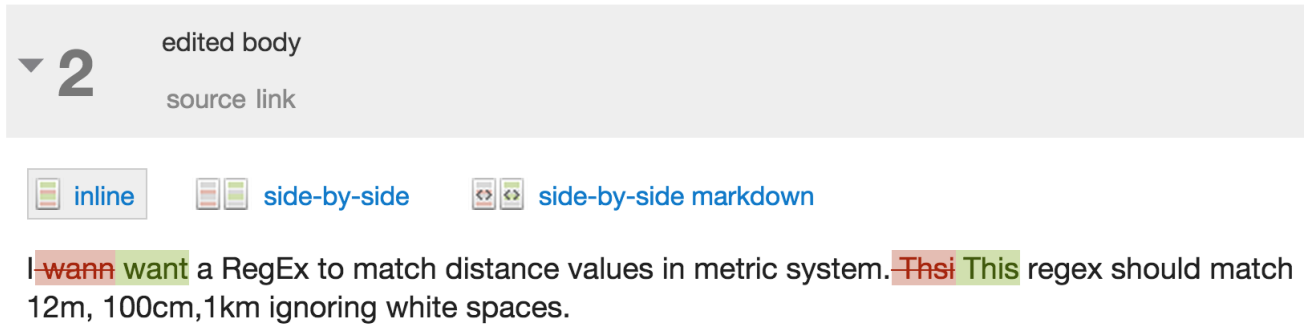

FIGURE 2: An Edits on Stack Overflow

Answers Any registered user can provide Answers to existing Questions ${ }^{10}$ A Question can have multiple Answers and the latter are ranked by total Votes.

Edits Registered users can also make or suggest minor changes to a Question or Answer: Edits. Edits help make the Questions and Answers more readable and understandable to future viewers 11

Votes Registered users can give up-votes or down-votes to Questions and Answers but not to Edits. Votes reward reputation points of the owner of a post: each up-vote on a Question gives the asker five points, whereas each up-vote on an Answer is worth ten points 12

\section{Theoretical Model Of User Contribution}

We propose a simple dynamic model of user contributions. Consider an infinite-period, discrete time line, and suppose agents discount the future according to the factor $\delta$. Each agent is an SO contributor and a job seeker. The agent's state space is limited to $s \in\{0,1\}$, where $s=0$ stands for current (or old) job and $s=1$ stands for future (or new) job. We assume $s=1$ is an absorbing state. To the extent this is not the case, our estimates of career concerns should be regarded as a lower bound of the real size of career concerns.

A fundamental hypothesis that we propose to test is that the probability of job transition - that is, the transition from $s=0$ to $s=1-$ is endogenous, specifically, a function of the agent's reputation $r_{t}$ :

$$
\mathbb{P}\left(s_{t}=1 \mid s_{t-1}=0\right)=p\left(r_{t}\right)
$$

is more likely to be done through Answers rather than Questions activity; second, the incentive structures between Questions and Answers/Edits are very different in that the former is done to seek help whereas the latter is to offer help (private contribution to online public goods). For more discussion please refer to Section 6.5

${ }^{10} \mathrm{~A}$ user can also answer his or her own question, but no reputation points are earned to avoid gaming the system.

${ }^{11}$ Most Edits correct grammar or spelling mistakes; clarify the meaning of a post; or add related information. Users with reputation under 2000 can suggest edits, which rewards them 2 points if accepted. Users with over 2000 reputation do not get the 2-point reward.

${ }^{12}$ Older Answers have more cumulative Votes. To control for the comparability among Answers given at different time, we measure the total Votes gained on each Answer within 30 days after an Answer was given. 
In each period, agents must decide how to allocate their time. We consider three types of tasks: Work, Answers and Edits tasks. Let $w_{t}, a_{t}$ and $e_{t}$ be the time devoted to each of these tasks, respectively. Each agent's time constraint is then given by

$$
w_{t}+a_{t}+e_{t}=T
$$

Consistently with the structure of $\mathrm{SO}$, we assume that $r_{t}$ is a function of past values of $a_{t}$ but not of past values of $e_{t}$. In fact, a crucial difference between Answers and Edits is that the former is a vote-generating activity whereas the latter is not ${ }^{13}$

We assume each agent's utility each period is additively separable between work task and SO-related tasks:

$$
u_{t}=g_{s}\left(w_{t}\right)+f\left(a_{t}, e_{t}\right)
$$

where $f(\cdot, \cdot)$ is a homothetic function and both $f(\cdot, \cdot)$ and $g(\cdot)$ are twice differentiable functions such that $f^{\prime}, g^{\prime}>0$ and $f^{\prime \prime}, g^{\prime \prime}<0$. The homotheticity of $f(\cdot, \cdot)$ means a constant marginal rate of substitution along rays, which implies that the time elasticity of $a$ and $e$ are the same, namely, $\eta_{a}=\eta_{e}$ where $\eta_{x}=\frac{d x / d T}{x / T}$. Many commonly used utility functions satisfy these assumptions, including constant elasticity of substitution (CES) functions where $f(a, e)=\left(\alpha a^{\rho}+(1-\alpha) e^{\rho}\right)^{\frac{1}{\rho}}$.

Notice we allow the utility from work to be state-dependent. In fact, the agent's demand for a new job results from our assumption that $g_{1}(w)>g_{0}(w)$.

In this model, $f\left(a_{t}, e_{t}\right)$ is the utility function derived from intrinsic motivations. The career incentive of $a_{t}$ activity is not included in the $f$ function, rather it enters through a higher $p\left(r_{t}\right)$ in order to transition to a job with higher value of $g(w)$.

Agents are forward looking: in each period $t$, they choose $w_{t}, a_{t}, e_{t}$ so as to maximize value $V_{t}(s)$, where $s=0,1$. The value functions are determined recursively as follows:

$$
\begin{gathered}
V_{t}(s)=\max _{w_{t}, a_{t}, e_{t}} g_{s}\left(w_{t}\right)+f\left(a_{t}, e_{t}\right)+\delta E V_{t+1}\left(s^{\prime}\right) \\
\text { subject to: } w_{t}+e_{t}+a_{t}=T
\end{gathered}
$$

Our main theoretical result is as follows:

\footnotetext{
${ }^{13}$ In addition to Answers, Questions can also attract votes (thus reputation points). However, most Questions are asked to solve work-related problems, and we consider them as part of $w$. Please refer to Section 6.5 for more discussion on Questions activity.
} 
Proposition 1. Suppose that $g_{0}(w)<g_{1}(w)$ and $g_{0}^{\prime}(w)<g_{1}^{\prime}(w)$. Then

$$
\left.a_{t}\right|_{s=1}<\left.a_{t}\right|_{s=0}
$$

Moreover,

$$
\left.\frac{a_{t}}{e_{t}}\right|_{s=1}<\left.\frac{a_{t}}{e_{t}}\right|_{s=0} \quad \text { iff } \quad p^{\prime}(\cdot)>0
$$

Proof: See the Online Appendix.

Proposition 1 establishes two effects of a job change: a decline in the time spent on providing Answers; and a decline in the relative time spent on Answers vis-à-vis Edits. The first effect (decline of Answers activity) can be decomposed into two effects: an increase in the marginal utility of time spent at work; and a decline in the utility of Answers due to diminished career incentives. Since there are two effects, a decline in Answers is a necessary but not sufficient condition for our career-concerns hypothesis. By contrast, the second effect takes place if and only if career concerns are present. It provides, therefore, a sharper test of our central hypothesis.

One advantage of a theoretical model is that it helps clarify the assumptions underlying an empirical identification strategy. The assumption that the Edits and Answers components in the utility function share the same elasticity with respect to changes in $T$ plays an important role. As individuals work for longer hours, the assumption is necessary to prevent Edits to respond disproportionally to changes in time availability. In Section 7, several tests are provided to test the validity of this assumption.

Proposition 2. Suppose that $p^{\prime}(\cdot)>0$ and $p^{\prime \prime}(\cdot)<0$. Then

$$
\left.\frac{a_{t}}{e_{t}}\right|_{s=0, r_{t-1}}>\left.\frac{a_{t}}{e_{t}}\right|_{s=0, r_{t-1}^{\prime}} \quad \text { for } \quad r_{t-1}<r_{t-1}^{\prime}
$$

Moreover, let $p(\cdot)$ take on more arguments and become $p\left(x_{t}, r_{t}\right)$. Assuming $\frac{d p\left(x_{t}, r_{t}\right)}{d a_{t}}>0, \frac{d p\left(x_{t}, r_{t}\right)}{d x_{t}}>0$, and $\frac{d^{2} p\left(x_{t}, r_{t}\right)}{d a_{t} d x_{t}}<0$. Then

$$
\left.\frac{a_{t}}{e_{t}}\right|_{s=0, x_{t}}>\left.\frac{a_{t}}{e_{t}}\right|_{s=0, x_{t}} \text { for } \quad x_{t}<x_{t}^{\prime}
$$

Proof: See the Online Appendix.

Proposition 2 shows the heterogeneous effects of career concerns on online activities for those with different online reputation and other characteristics (such as education, work experience, etc.), respectively. 
The first part of proposition 2 shows that if $p(\cdot)$ is a concave function, namely, marginal benefit of additional reputation on job offers is smaller for those already enjoying a good reputation, then the effect of career concerns would be smaller. Similarly, the second part of Proposition 2 says that the effect of career concerns would also be smaller if the marginal benefit of online reputation is smaller for those with better characteristics.

In Section 6, we will provide additional empirical tests, as well as discussions, on the heterogeneous effects by dividing the job seekers into groups based on their characteristics and provide more discussions.

\section{Data}

Our dataset is derived from the Stack Overflow (SO) and Stack Overflow Careers (SOC) sites.

SOC is a job matching website that hosts programming-related job listings as well as resumes of job candidates. For contributors, creating a resume on the website is free of charge but by invitation only; and the invitation is based on the contributors' recent activity to the site as well as their field of expertise ${ }^{14}$ On the resume, contributors can easily provide a link to their SO profile, through which employers can learn more about the job applicants' expertise: that is, potential employers observe the user's reputation score, a reflection of the quantity and quality of the user's contribution to SO.

Through a paid subscription, SOC helps employers by reducing their hiring search costs. First, SOC provides a select sample of high-level contributors invited by SO. Second, SOC includes a wealth of information regarding the job applicants' skill sets, including in particular their contribution history to SO. Finally, employers who access SOC may post their openings as well as search candidates by location, skills, and so on 15

We focus on a set of users that satisfy a series of criteria required by our empirical test: ${ }^{16}$ Located in the U.S. and Canada; job switchers (moving from one job to another); active users (at least one Answer and one Edit within the four-month period around a job change); profiles with links to SO.

Applying this series of criteria results in a sample of 1301 users with 1520 job switches ${ }^{17}$ For each user in our sample, we associate their user resumes (which include dates of job changes) to user IDs on SO. With

\footnotetext{
${ }^{14}$ The exact criteria is not disclosed by SO. It is also possible to request an invitation on the website.

${ }^{15}$ As of October 24th, 2015, there are 1283 jobs on SOC, with 893 jobs located in the U.S. and Canada. The number is quite small compared to jobs on other popular employment websites such as Monsters.com and Indeed.com, where employers can post jobs free of charge.

${ }^{16}$ A detailed account of the selection procedure can be found in the Online Appendix. It also includes a more detailed comparison and discussion on differences between SO users, SOC users, and our selected sample.

${ }^{17}$ Obviously, the sample we use is not representative of the whole population, since the majority of users have very few contribution activities. However, we do think it is a representative sample of active contributors.
} 
TABLE 1: DESCRIPTIVE STATISTICS

\begin{tabular}{lrrrrr}
\hline & Mean & Median & Std. Dev. & Min. & Max. \\
\hline User Activity (Monthly) & & & & & \\
Answers & 4.055 & 0 & 12.310 & 0 & 417 \\
Votes (from Answers) & 5.967 & 0 & 23.023 & 0 & 966 \\
Questions & 0.637 & 0 & 1.933 & 0 & 58 \\
Edits & 1.748 & 0 & 9.883 & 0 & 689 \\
User Characteristics & & & & & \\
Profile Views & 359.723 & 71.5 & 2170.283 & 0 & 112967 \\
Total UpVotes & 334.669 & 82 & 800.728 & 0 & 15143 \\
Reputation Points & 1603.965 & 150 & 6204.839 & -6 & 132122 \\
Age & 33.889 & 33 & 7.433 & 16 & 95 \\
Time on SO & 4.225 & 4.337 & 1.503 & 0.167 & 6.507 \\
\hline
\end{tabular}

the user IDs at hand, we then collect their activities on SO.

Table 1 provides some descriptive statistics of SO activities from the sample of 1301 users. A typical SO user is not active in writing Questions or Answers. The activity distributions are fairly right-skewed, suggesting that a few users are disproportionally responsible for much of the content created on SO. The lower portion of the table suggests that typical users of SO are in their early 30s and have been on SO for 4 years.

\section{Identification Strategy}

Conceptually, our identification strategy is straightforward: job seekers are active on SO to signal their ability and thus obtain a better job. If career concerns are important to incentivize user activity, then we expect a drop in such activity once the goal (a better job) is attained. Since career aspirations may not be satisfied by a single advancement, career concerns might not entirely disappear; but at least they are diminished at the start of a new job.

In practice, there are various confounding factors that make measurement of career-concern effects difficult. In particular, a reduction in online activity following a job change may simply result from a reduction in time availability: a new job often requires training and familiarization with a new environment. In fact, as the first part of Proposition 1 states, we expect a drop in $a_{t}$ through two effects: a drop in career concerns (measured by $p\left(r_{t}\right)$ in the model); and an increase in work activities (measured by the shift from $g_{0}(w)$ to $g_{1}(w)$ in the model).

To account for these effects, we use the differential change in Answers relative to Edits to test the 


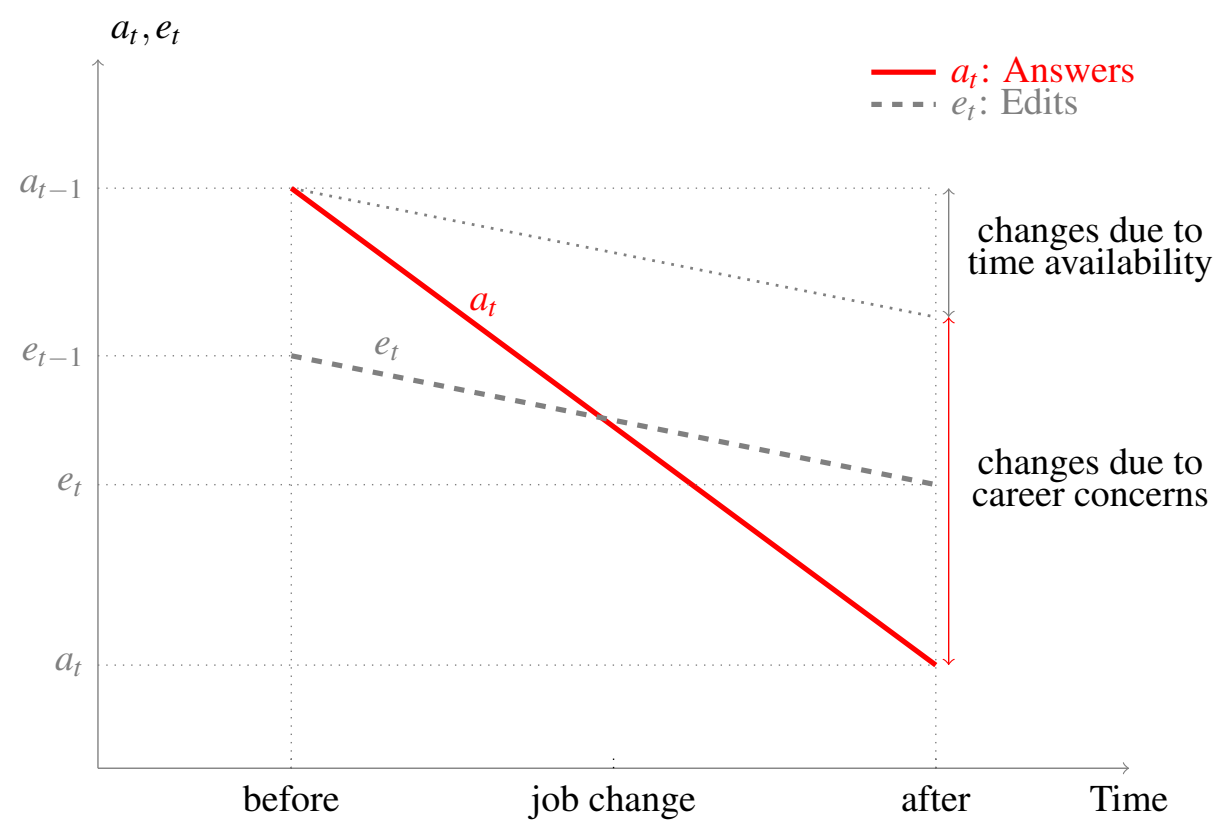

FIGURE 3: Graphical Illustration of Identification Strategy: Difference-in-Differences

Notes: Treatment group: Answers activity (a); Control group: Edits activity (e). All activity data comes from the same sample of contributors. DD coefficient is calculated as $\left(a_{t}-a_{t-1}\right)-\left(e_{t}-e_{t-1}\right)$, which measures the differences of Answers-Edits gap before and after a job change.

hypothesis that Answers are motivated by career concerns. A crucial difference between Edits and Answers is that the latter give rise to Votes, whereas the former do not. Therefore, we expect Answers to decline by more than Edits after individuals switch jobs. Our DD approach assumes that, aside from changes in job status, Edits and Answers follow a parallel path. Since this is such a crucial assumption, in Section 7 we provide supporting evidence.

Essentially, our DD approach corresponds to the second part of Proposition 1 . Figure 3 illustrates the main idea: after starting a new job, the reduction in Answers activity results from two effects: career concerns and time availability (or, opportunity cost of work time); however, the reduction in Edits activity results exclusively from the time availability effect; therefore, the difference between the changes in Answers and in Edits identifies the effect of job change on career-concerns incentives for Answers.

Figure 4 provides preliminary evidence regarding our hypothesis. It plots the monthly average of the logarithm of user activities in a 20 -month window centered around a contributor's job change event. Both Answers and Edits activity experience a significant drop when a user starts a new job (month 1); however, the drop in Answers activity is considerably larger than the drop in Edits activity.

Naturally, several other alternative hypotheses may explain these dynamics. In Section 7, we present and evaluate several hypotheses under which the parallel trend assumption could be violated, and evaluate 


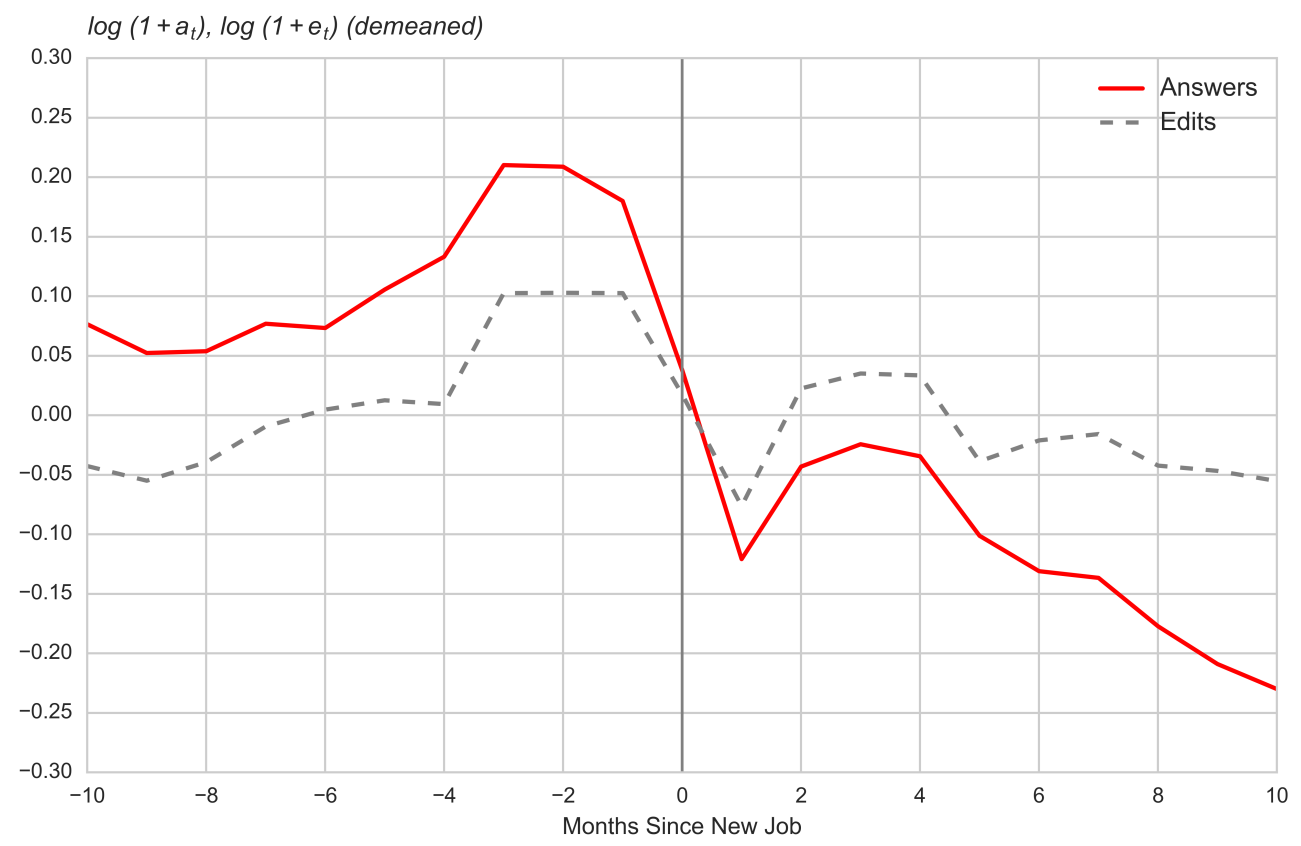

FIgURE 4: Average Monthly Activity on Stack Overflow (Answers and Edits)

Notes: $\mathrm{x}$-axis: Number of months since a new job starts. $t=1$ means the first month of a new job. People with different starting dates are normalized to the same timeline based on number of months since the new job. $y$-axis: $\log$ differences of activities.

the validity of each hypothesis.

\section{Empirical Analysis}

We now come to a more formal test of the hypothesis implied by Proposition 1 . Our empirical analysis focuses on the sample of 1,301 users who were subject to 1,520 job switches during the November 2008November 2014 period. For each of these job switches, we measure activity levels by activity type and by month. Specifically, define period 1 as the month when a job change takes effect (that is, the month users start a new job as listed on their). We then consider 3 months prior to a job switch $(-3,-2,-1)$; and 3 months subsequent to a job switch $(+2,+3,+4)$. We exclude months 0 and 1 ; in this way we get a clearer perspective on the periods before and after the job change without contaminating the data with noise stemming from the process of job change.

\subsection{Empirical Specification}

As illustrated in Figure 3 and 4, our identification strategy is derived from a difference-in-differences approach. However, instead of comparing the behavior of different individuals, we focus on the same set of 
individuals and compare their behavior across different activities before and after a job change:

$$
y_{i j t}=\alpha_{i j}+\beta S_{i t}+\gamma J_{j} S_{i t}+\lambda_{j t}+X_{i j t} \theta+\varepsilon_{i j t}
$$

In regression 5, the dependent variable $y_{i j t}$ includes two different types of online activities, including one votes-generating activity (VGA) and one non-VGA, which are indicated by subscript $j$. VGAs can be Answers $(j=a)$, Votes gained from Answers $(j=v)$, or Questions $(j=q)$; non-VGA is Edits $(j=e)$. All the activities are measured in logarithms. One advantage of this approach is that the coefficients can be readily interpreted as percent variations. $S_{i t}$ is the state dummy variable: $S_{i t}=0$ corresponds to the periods before a job change takes place for user $i$, whereas $S_{i t}=1$ corresponds to the periods after a job change takes place. $J_{j}$ is a dummy variable that takes on value 1 if the activity is a $\operatorname{VGA}(j=a, v, q)$ and 0 otherwise $(j=e)$.

$\alpha_{i j}$ are individual fixed effects for each type of activity, which can control for many individual characteristics that could influence online contribution levels, such as ability, personal preference, gender, age, etc. The fixed effects are added at both individual and activity level due to contributors' preference of one task over another. For example, some contributors ask many Questions but rarely give any Answers.

$\beta$ measures changes in Edits activity before and after a job switch. The main parameter of interest is the DD coefficient $\gamma \cdot \gamma$ measures the additional change in a VGA (Answers or Questions) over the changes to a non-VGA (Edits) after a job change.

The two parts of Proposition 1 can be expressed by the regressions coefficients $\beta$ and $\gamma$. Specifically, we expect the level of SO activity to drop subsequently to a job shift, that is, we expect $\beta$ and $\beta+\gamma$ to be negative. Moreover, we expect the drop in Answers to be greater than that of Edits, so that $\gamma<0$, in addition to $\beta<0$.

Seasonality and Duration Effects. In order to obtain a more accurate estimate of the effect of career concerns, we include additional activity data from a large sample of 96k active SO users, which can control for variations due to seasonality and duration effects 18

Online contribution might be more active in certain months than others; job changes can also occur more often in certain months of the year. We include additional year and month dummies to control for such potential effects, denoted by $\lambda_{j t}$ in regression 5. Duration effects include the initial excitement of discovering SO, which can change over time and have heterogeneous effects on Answers and Edits activity.

\footnotetext{
${ }^{18}$ Although without information on $\mathrm{CV}$ and job status, we observe their online activity over time. The additional data is used to control for seasonality and duration effects only.
} 
TABLE 2: EfFects Of Career Concerns On Answers And Edits Activity

\begin{tabular}{|c|c|c|c|c|}
\hline & \multicolumn{2}{|c|}{ Panel A: $y \in\{$ Answers, Edits $\}$} & \multicolumn{2}{|c|}{ Panel B: $y \in\{$ Votes, Edits $\}$} \\
\hline & (1) & (2) & (3) & (4) \\
\hline $\operatorname{NewJob}(S)$ & $\begin{array}{c}-0.0738 * * * \\
(0.019)\end{array}$ & $\begin{array}{c}-0.0742 * * * \\
(0.019)\end{array}$ & $\begin{array}{c}-0.0738 * * * \\
(0.019)\end{array}$ & $\begin{array}{c}-0.0742 * * * \\
(0.019)\end{array}$ \\
\hline NewJob $(S) \times$ Answer $/$ Vote $(J)$ & $\begin{array}{c}-0.1627 * * * \\
(0.033)\end{array}$ & $\begin{array}{c}-0.1236 * * * \\
(0.033)\end{array}$ & $\begin{array}{c}-0.1943 * * * \\
(0.037)\end{array}$ & $\begin{array}{c}-0.1536 * * * \\
(0.037)\end{array}$ \\
\hline Seasonality dummy & & $\mathrm{x}$ & & $\mathrm{x}$ \\
\hline Duration dummy & & $\mathrm{x}$ & & $\mathrm{x}$ \\
\hline Contributors & 1301 & 97723 & 1301 & 97723 \\
\hline$N$ & 18192 & 9105862 & 18192 & 9105862 \\
\hline$R^{2}$ & 0.014 & 0.033 & 0.014 & 0.027 \\
\hline
\end{tabular}

Notes: Robust standard errors in parentheses, clustered at the level of the individual-activity type level. $* * * p<0.01$, ** $p<0.05, * p<0.1$

We measure duration as the count of the number of months since the first activity on SO for each user and include dummies for all distinct values of duration, denoted by $D_{i j t}$ in regression 5 . A separate set of seasonality and duration dummies is added for each type of activity, in order to control for the heterogeneous effects of seasonality and duration on different activities.

\subsection{Main Effects of Career Concerns}

Table 2 presents our core results. The results are organized into two panels, using the number of Answers and Votes gained from Answers as measures of Answers activity. For each panel, the first regressions (columns 1 and 3) shows our base results without controlling for seasonality and duration effects. We thus have 18,192 observations (1,516 job switches from 1,301 contributors times 6 months: three prior to the job switch, three subsequent to the job switch, and times 2 activities: Answers and Edits). The second regressions in each panel (columns 2 and 4) shows the results while controlling for seasonality and duration effects, using activities from a large sample of SO users.

Column 1 shows that after switching to a new job, Edits activity experiences a significant drop of $7.38 \%$. Moreover, the DD coefficient shows an additional drop of 16.27\% in Answers activity, which we attribute to career concerns. The total changes in Answers activity can be calculated by $-7.38 \%-16.27 \%=-23.65 \%$. The results confirm the predictions from Proposition 1 that both coefficients are negative. Column 2 adds a set of dummies that control for seasonality and duration effects. This reduces our estimate of the treatment effect only slightly, to a statistically significant $12.36 \%$ decline.

Columns 3 and 4 report the same set of estimates using Votes instead of Answers to measure the vote- 
generating activity. Votes is a measure that includes both quantity and quality of Answers, and it can be a better measure of the amount of effort in contribution activities. The results using Votes give similar but slightly larger estimates than results using Answers. In Section 6.4, we investigate in depth the impact of increased effort on quantity and quality of Answers.

\subsection{Month-to-Month Comparison}

Table 2 summarizes DD estimates by comparing the differential changes of Answers and Edits activity in the 3-month period before and after a job change. We also explore the effects of career concerns over a longer period of time. Using period -2 as the baseline period, we compare the activity of all other periods to period $-2{ }^{19}$ We also control for seasonality and duration effects using the same $96 \mathrm{k}$ SO users mentioned before. We do so by estimating two following specifications:

$$
\begin{aligned}
y_{i t} & =\alpha_{i}+\sum_{\tau=-20}^{20} \beta_{\tau} \mathbb{1}\left(P_{i t}=\tau\right)+\lambda_{t}+X_{i t} \theta+\varepsilon_{i t} \\
y_{i j t} & =\alpha_{i j}+\sum_{\tau=-20}^{20}\left(\beta_{\tau} \mathbb{1}\left(P_{i t}=\tau\right)+\gamma_{\tau} J_{j} \mathbb{1}\left(P_{i t}=\tau\right)\right)+\lambda_{j t}+X_{i j t} \theta+\varepsilon_{i j t}
\end{aligned}
$$

Regression 6 measures how each activity vary over time relative to baseline period -2 , which is denoted by $\beta_{\tau}$. Regression 7 estimates the differential changes between a VGA (i.e. Answers) and non-VGA (i.e. Edits) between the baseline period -2 and all other periods, and the DD coefficient is denoted by $\gamma_{\tau}$.

$\lambda_{j t}$ and $X_{i j t}$ control for seasonal and duration effects for each type of activity. $P_{i t}$ represents the number of months after a job change, and $\mathbb{1}\left(P_{i t}=\tau\right)$ is a dummy variable which equals to 1 if the month $t$ for user $i$ corresponds to $\tau$ months after a job change.

Figure 5 plots the demeaned values of the estimates of $\beta_{\tau}$ for Answers and Edits activity 20 It is essentially Figure 4 with seasonality and duration effects removed. Answers and Edits activity remain relatively stable from 20 to 5 months before the event of a job change. During the 5-month period before a job change, both Answers and Edits activity experience a rapid increase, with Answers growing more than Edits. Then there is a rapid drop in both Answers and Edits activity starting from one month before a job change, with Answers decreasing significantly more than Edits and both continue to decrease over time.

Figure 6 shows the differential changes in Answers and Edits activity over time by plotting the DD estimates $\gamma_{\tau}$ for $\tau \in[-20,20]$, as well as the $95 \%$ confidence interval. Before switching to a new job, all the DD estimates are negative but not significantly different from zero. Following the job change event, all the

\footnotetext{
${ }^{19}$ Period -2 is used as the baseline period since it has the highest average Answers activity level.

${ }^{20}$ The detailed estimates of $\beta_{\tau}$ from regression 6 and $\gamma_{\tau}$ from regression 7 can be found in the Online Appendix.
} 


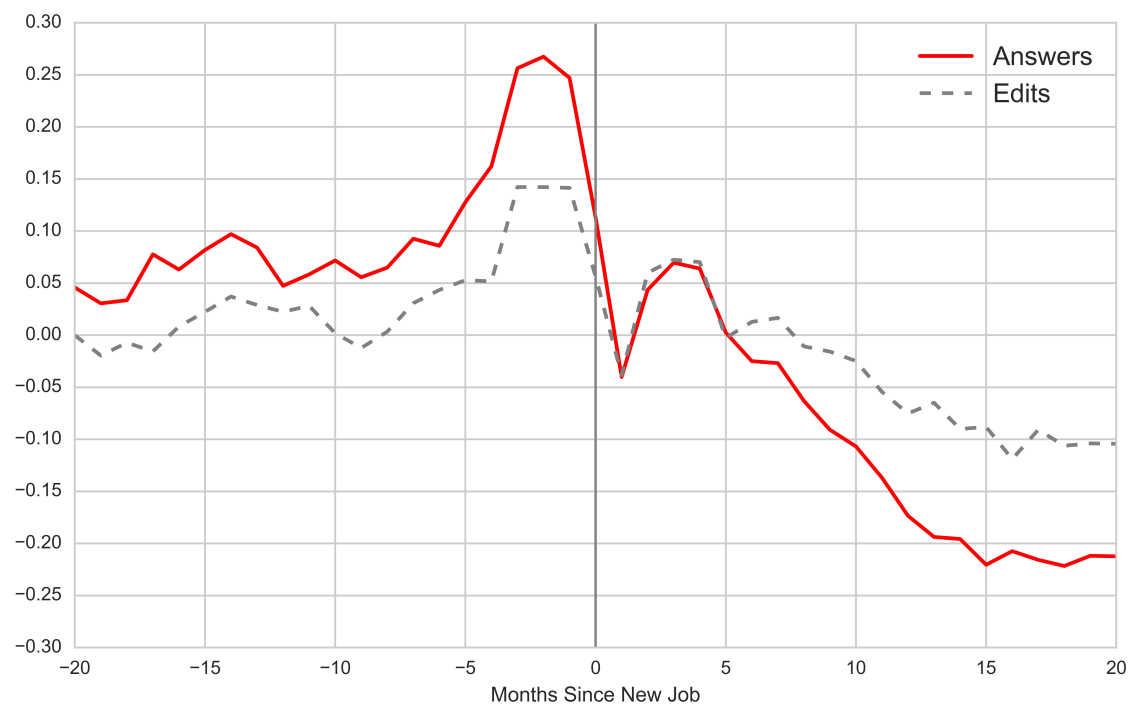

FIGURE 5: Activity Trend (After Controlling for Seasonality and Duration Effects)

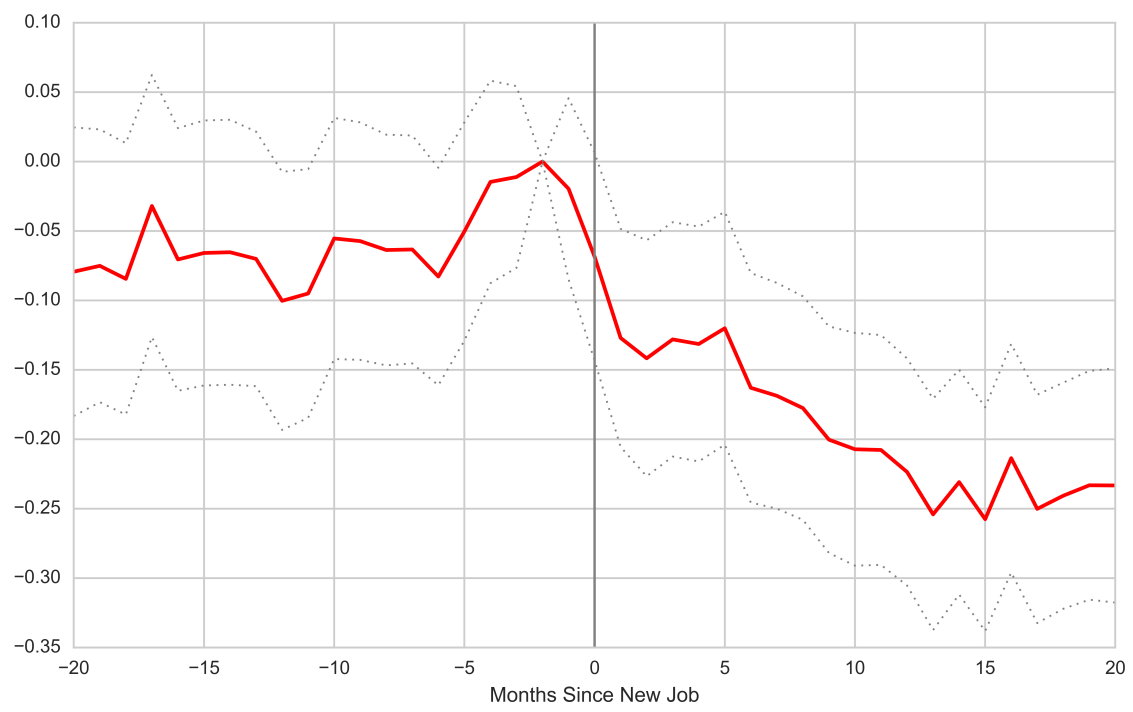

FIgURE 6: Differences Between Answers and Edits Activity With 95-Percent Confidence Interval 


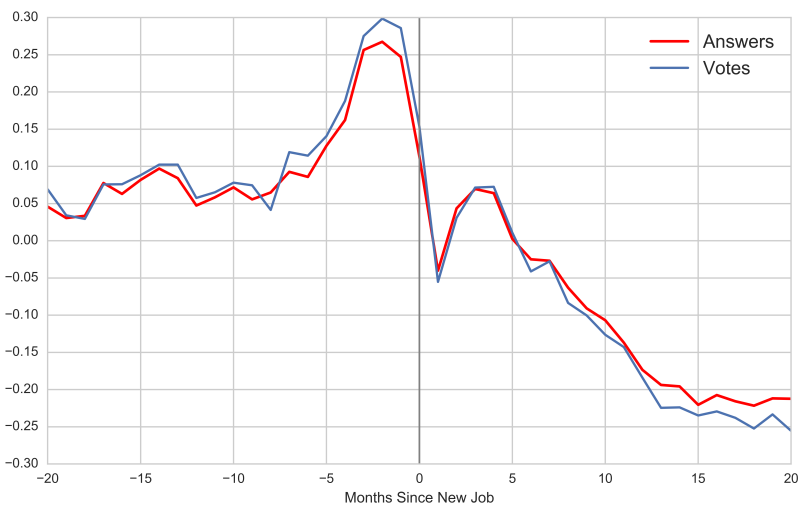

(A) Number of Answers vs. Votes received from Answers

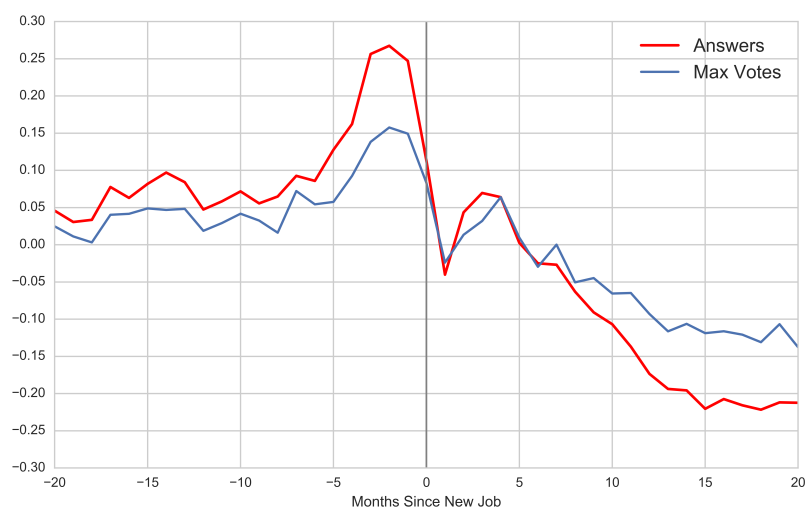

(B) Number of Answers vs. Votes from a best Answer

FIGURE 7: Quantity and Quality of Answers Activity

DD estimates are significantly negative, i.e. Answers decrease significantly more than Edits.

Both figures illustrate that the Answers activity continues to drop over time after a job change. This may be explained in several ways. First, the first few months are often considered as probationary periods where both employers and employees can freely terminate contracts. Thus career concerns drop significantly but do not completely disappear as both parties need time to determine the match quality. If this is the case, then our results from regressions 6 and 7 underestimate the effect of career concerns. Second, job seekers may form the habit of contributing to SO as they improve their online reputation, which can have long term effects on contribution activities. Without taking into account habit formation, our DD estimates provides a lower bound of the true effect of career concerns.

\subsection{Signaling Game: Quality vs Quantity}

The classic career-concerns hypothesis in Holmström (1982/99) shows that job seekers exert effort to signal their unobserved ability. Questions to ask are (a) what are job seekers signaling through SO, and (b) what information do employers obtain from the online activity of a job candidate?

Figure 7A plots the trend of Votes and Answers over time. The correlation between the two measures is remarkably high. The fact that the average quality of Answers remains constant seems to contradict the basic intuition of the career concerns story. However, one cannot conclude that career concerns have no effects on the quality of Answers. Given a fixed supply of Questions, the additional efforts to answer Questions should lead to both better Answers from Questions a contributor would answer regardless of career concerns, and more Answers from Questions a contributor would not answer without career concerns due to low matching qualities. Thus one should observe that as the time of a job change approaches, a job seeker gives more 
Answers, and at the same time, the qualities of the Answers are mixed.

To test this hypothesis, we pick the best Answer (measured by Votes) given by a contributor for each month, and Figure 7B plots the average Votes from the best Answers over time. It shows that the quality of best Answers follows a similar pattern to the number of Answers. This is consistent to the hypothesis that apart from quantity of Answers, contributors also improve the quality of Answers before a job change. However, caution should be taken regarding the causality, since the result can also be explained by the random distribution of matching quality, with which the largest order statistic (Max Votes) increases with a larger sample (number of Answers).

\subsection{Questions Activity}

Questions is another important activity on SO, since there are no Answers without Questions. Here we investigate how Questions activity changes surrounding the event of a job change. The plotting of Questions activity (included in the Online Appendix) shows little changes in the number of Questions over time. Questions activity reduces slightly at the end of the old job and then increases right after the starting month of a new job. Questions activity is a learning tool; and a shift to a new job creates new learning demands even for new jobs with the same set of technology, an effect that seems to compensate for the higher opportunity cost of time spent on SO as well as the diminished incentive to build a reputation. It is also possible that asking Questions might be perceived as inability to solve problems, therefore job seekers avoid asking Questions 21

\subsection{Heterogeneous Effects of Career Concerns}

The hypothesis of career concerns states that job seekers make efforts to improve the signals to employers that reflect ability. Such signals in the real world can be multi-dimensional, and Proposition 2 implies heterogeneous responses by job seekers with different backgrounds. This subsection tests the prediction by comparing the reactions of job seekers by reputation and education levels.

Part 1 of Proposition 2 shows that since reputation points on SO are cumulative, signals are carried through both existing and new Answers activities. When pursuing new employment opportunities, job seekers with different levels of reputation might have heterogeneous responses to career incentives. For a job seeker who already enjoys an outstanding reputation on SO, the marginal benefit of extra effort to improve that signal should be relatively small. We associate each job switch with the reputation points at the time of the switch, and conduct separate analysis by splitting the sample into four equal groups of job

\footnotetext{
${ }^{21}$ Please see the Online Appendix for more analysis related to Questions activity.
} 
TABLE 3: EFFects Of CAREer Concerns By EducATIOn LEVEls

\begin{tabular}{|c|c|c|c|c|c|c|c|c|}
\hline & \multicolumn{4}{|c|}{ Panel A: Reputation Quartiles } & \multicolumn{4}{|c|}{ Panel B: Education Level } \\
\hline & (1) & (2) & (3) & (4) & (5) & (6) & (7) & (8) \\
\hline & Rep.Q1 & Rep.Q2 & Rep.Q3 & Rep.Q4 & HS & College & Masters & $\mathrm{PhD}$ \\
\hline \multirow[t]{2}{*}{ NewJob } & $0.157 * * *$ & $-0.067^{*}$ & $-0.166^{* * *}$ & $-0.251 * *$ & 0.102 & $-0.084 * * *$ & -0.045 & -0.036 \\
\hline & $(0.04)$ & $(0.03)$ & $(0.04)$ & $(0.04)$ & $(0.16)$ & $(0.03)$ & $(0.05)$ & )) \\
\hline \multirow[t]{2}{*}{ NewJob $\times$ Answer } & 0.102 & $-0.228 * * *$ & $-0.242 * * *$ & $-0.132 *$ & -0.205 & $-0.121 * * *$ & $-0.164 * *$ & -0.088 \\
\hline & $(0.06)$ & $(0.06)$ & $(0.07)$ & $(0.07)$ & $(0.25)$ & $(0.04)$ & $(0.08)$ & $(0.17)$ \\
\hline Contri & 356 & 350 & 340 & 311 & 12 & 778 & 230 & 51 \\
\hline Contributors (ctrl) & 96422 & 96422 & 96422 & 96422 & 96422 & 96422 & 96422 & 96422 \\
\hline$N$ & 9092012 & 9092002 & 9092012 & 9092000 & 8944282 & 8955016 & 8947346 & 8944870 \\
\hline$R^{2}$ & 0.033 & 0.033 & 0.033 & 0.033 & 0.033 & 0.033 & 0.033 & 0.033 \\
\hline
\end{tabular}

Notes: Reputation Points: Min: 0; First Quartile: 770; Median: 2,124; Third Quartile: 5,265; Max: 132,067. Seasonality and duration dummies are included in all regressions. Robust standard errors in parentheses, clustered at the level of the individual-activity type level. $* * * p<0.01, * * p<0.05, * p<0.1$

switches. Panel A in Table 3 shows that job seekers in the second and third quartiles (columns 2 and 3) respond most to career incentives at $22.8 \%$ and $24.2 \%$. Those with highest reputations (column 4) show a smaller effect at $13.2 \%$. The most striking result comes from job seekers with lowest SO reputations. The estimate has an opposite sign compared to results from other groups: one possible explanation is that low reputation users probably do not provide a link to their SO profile when applying for jobs.

Part 2 of Proposition 2 predicts that job seekers with better offline signals respond less to career concerns through online activities. We test this by looking at the highest education levels achieved by the job seekers. An individual without post-secondary education should have more incentive to improve the signal through other activities, including online activity; an individual with an advanced degree probably relies less on online activity to signal ability. We extract the highest degree obtained by SOC users and divide them into four groups: High School (HS), Four-Year or Community College (College), Masters and Ph.D. Then we conduct separate analyses for each group. As shown in Panel B in Table 3 , the magnitude of the DD estimates is roughly consistent to the hypothesis of career concerns. Those with a high school diploma as their highest education respond to a job change the most (-20.5\% in column 1$)$, though insignificant given a very small sample size; on the other hand, those with a $\mathrm{Ph} . \mathrm{D}$. degree show the least response to job switches $(-8.8 \%$ in column 4$)$.

Proposition 2 also predicts those with more work experience might respond more to changes in career incentives ${ }^{22}$ As such, we expect our main result (job change leads to less contribution) to be lower for more experienced agents. We investigate this assumption using age inferred from information on CV and find the

\footnotetext{
${ }^{22}$ Holmstrom (1982) also predicts that as a career proceeds, the market is better informed about a worker's ability and the incentives to signal such ability are lower.
} 
opposite results. This could result from very noisy measures of age. More likely, it results from confounding effects: a "young" worker has more to gain from signaling quality, which would suggest a stronger effect; but for a "young" worker a job change is just the first of many and the incentives to signal are likely to remain high, which in turn suggests a lower drop in incentives. Another possible explanation is that career incentive plays a more important role in driving voluntary contribution for younger users compared to the older ones, a pattern consistent to what we usually observe in other real-world volunteer activities.

\section{Testing Identification Assumptions}

Our identification relies fundamentally on the parallel trends assumption in Answers and Edits activity. That is to say, if a job switch did not occur (i.e. without a change in career incentives), the relative ratio of Edits and Answers would have remained constant. Since this assumption plays a central role in our identification strategy, additional evidence on it is warranted. In this section, we first provide some evidence to support this assumption. Then we will discuss and test for several major challenges to the assumption.

\subsection{Evidence of Parallel Trends: Plotting of Online Activities}

Figure 4 plots the average logged activity over time. It provides some evidence on the parallel changes of Answers and Edits over time. Figure 5 further plots the same activities, while removing the potential confounding effects from seasonality and duration. In the periods further away from a job change, the level of career incentives should be relatively stable. Figure 5 shows that after period 10 , although activity levels vary, Answers and Edits move similarly over time. This supports for the parallel trend assumption.

\subsection{Evidence of Parallel Trends: Within-Job Activity}

The parallel trend assumption implies that if there were no changes in career incentives, then variations in time availability should have similar effects on Answers and Edits activity. To show cleaner evidence, we identify a period of stable employment for each contributor. We assume that during these periods, though a contributor's availability fluctuates, the change in career concerns is small compared to what we observe leading up to a job change. Consistent with our basic identifying assumption, we expect the differences between $a_{t}$ and $e_{t}$ to remain constant.

Figure 8 shows the values of Answers and Edits for months 5 to 42 after a job change. Consistent with our underlying assumption, the differences between the two are fairly constant. 


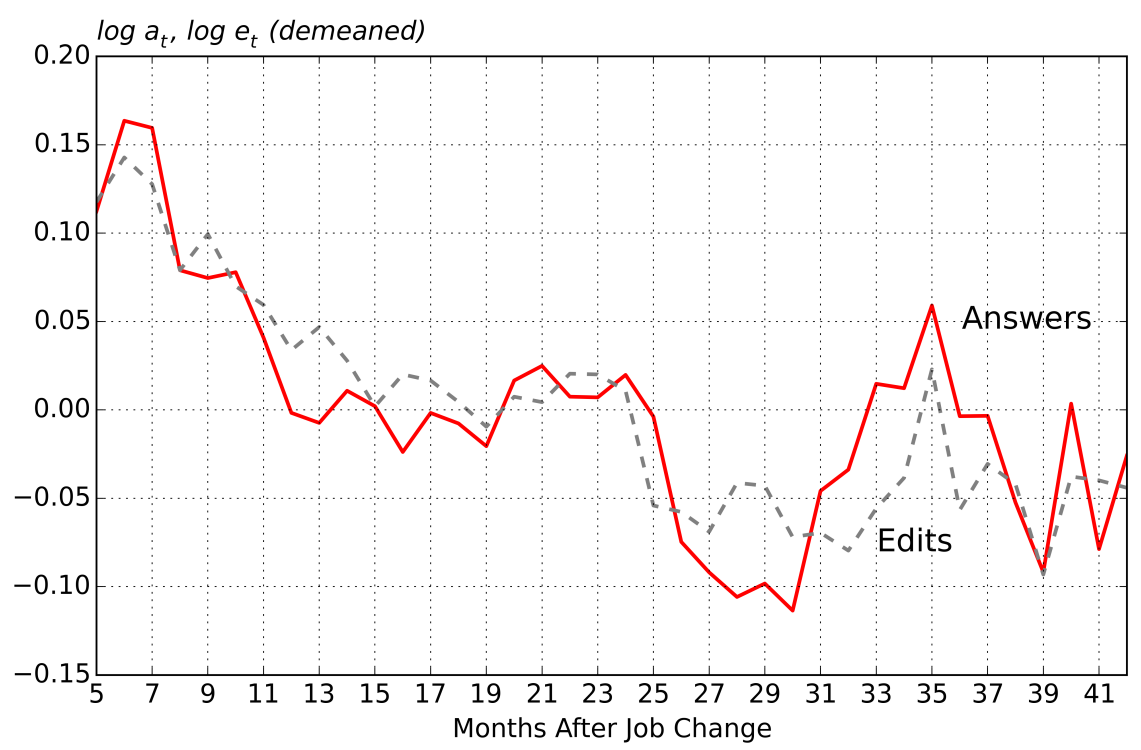

FIgURE 8: Within-Job Variations of Answers and Edits Activity

\subsection{Integer Constraints}

The validity of the career-concerns hypothesis relies on the assumption that changes in time availability due to a busier work schedule affect Answers and Edits activity similarly. However, an alternative interpretation that can explain our previous result that $a_{t} / e_{t}$ drops subsequent to a job change is that users are faced with an "Integer Constraint:" 23 Answers activity requires higher set-up costs than Edits. When job changes reduce availability, contributors have less time to allocate to Answers; Edits typically require less time and are easier to fit into a busy schedule.

The rich dataset of SO user activity along with employment history of SOC users, allows us to test whether the integer-constraint problem exists in our study and to what extent (i.e. whether we can reject the hypothesis of career concerns).

1. Weekdays vs. Weekend Activities. Following the start of a new job, a full schedule should reduce user availability predominantly during weekdays rather than weekends. Accordingly, we split our sample into weekday and weekend activities and conducted separate DD analyses. To the extent that work hours are more concentrated on weekdays, the integer-constraint hypothesis should have a more significant effect on $a_{t} / e_{t}$ during weekdays.

Table 4 shows the results of the DD regressions split into weekdays and weekends. Panel A and B uses Answers and Votes as measures of reputation-generating activities. If there were no integer constraints and

\footnotetext{
${ }^{23}$ Integer Constraint originally comes from mathematical programming when some choice variables are restricted to integer values. With this constraint, the agent has less freedom to allocate resources.
} 
TABLE 4: EFFects Of Job Changes On WeEkday Vs. WeEKend ACTIVIties

\begin{tabular}{lccccc}
\hline & \multicolumn{2}{c}{ Panel A: $y \in\{$ Answers, Edits $\}$} & & \multicolumn{2}{c}{ Panel B: $y \in\{$ Votes, Edits $\}$} \\
\cline { 2 - 3 } & $(1)$ & $(2)$ & & $(3)$ & $(4)$ \\
& Weekday & Weekend & & Weekday & Weekend \\
\hline NewJob & $-0.072^{* * *}$ & $-0.060^{* *}$ & & $-0.072^{* * *}$ & $-0.060^{* *}$ \\
& $(0.02)$ & $(0.02)$ & & $(0.02)$ & $(0.02)$ \\
NewJob $\times$ Answer $/$ Vote & $-0.122^{* * *}$ & $-0.103^{* *}$ & & $-0.135^{* * *}$ & $-0.124^{* * *}$ \\
& $(0.04)$ & $(0.04)$ & & $(0.04)$ & $(0.05)$ \\
\hline Contributors (DD) & 1159 & 374 & & 1159 & 374 \\
Contributors (ctrl) & 96422 & 51296 & & 96422 & 51296 \\
$N$ & 7378770 & 2895384 & & 7378770 & 2895384 \\
$R^{2}$ & 0.031 & 0.043 & & 0.026 & 0.031 \\
\hline
\end{tabular}

Notes: Seasonality and duration dummies are included in all regressions. Robust standard errors in parentheses, clustered at individual-activity type level. $* * * p<0.01, * * p<0.05, * p<0.1$

all users contribute to Answers and Edits activity on both weekdays and weekends, then there would be no differences between the DD estimates from weekday and weekend activities ${ }^{24}$ Broadly speaking, the coefficient estimates are similar to those in the base model, and the difference between the estimates using weekday and weekend activities is relatively small.

The difference between the DD estimates from weekday and weekend activities implies that although the integer-constraints problem might exist for certain users, it does not explain the additional drops in Answers relative to Edits after a job change. It fails to reject the career-concerns hypothesis.

2. Internal Promotion. Internal promotion is an important case in two ways: First, a promotion often assumes more managerial duties that lead to more significant changes in work schedule than lateral moves. In this way, internal promotion is most likely to satisfy the integer-constraint hypothesis. Second, the hypothesis of career concerns states that a job seeker signals to potential employers through online activity due to employers' inability to accurately access job candidates based on limited information. That is unlikely in the case of an internal promotion, since past internal performance is transparent to the employer. ${ }^{25}$

Our analysis includes both weekday and weekend activities. If an integer-constraint problem exists for job seekers who received internal promotions, then for weekday activities, we expect to observe an additional reduction in Answers relative to Edits. However, an internal promotion should affect weekday work schedule

\footnotetext{
${ }^{24}$ The majority of contribution activities take place on weekdays rather than on weekends. The selection requirement of having at least one Answers and Edits activity leads to a smaller sample of users for the analysis of weekend activities.

${ }^{25}$ It is conceivable that a job seeker might pursue outside opportunities in order to bargain with a current employer. In this case, public signals become valuable for internal promotions. Unfortunately, we do not have data on how promotions occur. However, we do not think most employees being reviewed for promotion use this bargaining tool. Otherwise, firms might establish internal policies that forbid employees from building high-quality public signals.
} 
TABLE 5: EFFEcts Of Job Changes For Internal Promotions

\begin{tabular}{|c|c|c|c|c|c|c|}
\hline & \multicolumn{2}{|c|}{ Panel A: All } & \multicolumn{2}{|c|}{ Panel B: Same Company } & \multicolumn{2}{|c|}{ Panel C: Promotion } \\
\hline & (1) & (2) & (3) & (4) & $(5)$ & (6) \\
\hline & Weekday & Weekend & Weekday & Weekend & Weekday & Weekend \\
\hline \multirow[t]{2}{*}{ NewJob } & $-0.072 * * *$ & $-0.060 * *$ & -0.070 & -0.039 & $-0.151 * *$ & -0.157 \\
\hline & $(0.02)$ & $(0.02)$ & $(0.05)$ & $(0.08)$ & $(0.06)$ & $(0.10)$ \\
\hline \multirow[t]{2}{*}{ NewJob $\times$ Answer } & $-0.122 * * *$ & $-0.103 * *$ & -0.090 & -0.119 & -0.156 & -0.008 \\
\hline & $(0.04)$ & $(0.04)$ & $(0.09)$ & $(0.13)$ & $(0.11)$ & $(0.19)$ \\
\hline Contributol & 1159 & 374 & 142 & 41 & 80 & 21 \\
\hline Contributors (ctrl) & 96422 & 51296 & 96422 & 51296 & 96422 & 51296 \\
\hline$N$ & 7378770 & 2895384 & 7364466 & 2890896 & 7363686 & 2890644 \\
\hline$R^{2}$ & 0.031 & 0.043 & 0.031 & 0.043 & 0.031 & 0.043 \\
\hline
\end{tabular}

Notes: Seasonality and duration dummies are included in all regressions. Robust standard errors in parentheses, clustered at individual-activity type level. $* * * p<0.01, * * p<0.05, * p<0.1$

only, leaving the job seeker similar levels of freedom to organize her schedule on the weekends. Therefore, we expect not to observe a differential effect of an internal promotion on Answers and Edits activities on the weekend.

Table 5 summarizes DD estimates for both internal moves and internal promotions, using weekday and weekend activities separately. Panel A uses all contributors for comparison purposes, which has the same results as Panel A (column 2 and 4) of Table 4 . Panel B focuses on internal moves, i.e. job changes within the same company. The estimates in Panel B are not vastly different from those in Panel A in magnitude but both become insignificant (most likely due to a smaller sample size). One potential concern is that many internal moves are lateral and do not necessarily require managerial duties. Panel $\mathrm{C}$ focuses on internal promotions using a stricter measure based on job title information. Column 5 shows that Answers experience an additional drop of $15.6 \%$ compared to Edits on weekdays. Although the estimate is insignificant (due to a small sample size), it supports the hypothesis of integer constraints for internally promoted workers. Column 6 shows a negligible DD estimate using weekend activities, which provides additional evidence that only weekday activities are affected by integer constraints.

To summarize, Panel $\mathrm{C}$ of Table 5 shows the likely presence of the integer-constraint problem in our DD analysis. Although this problem does not disprove the career-concerns hypothesis, it provides certain explanations regarding the different DD estimates from weekday and weekend activities in Table 4. 


\subsection{Skills Mismatch}

Another interpretation for the decrease in Answers following a job shift is that the new position requires different skills. For example, a C++ programmer may switch to a job that requires knowledge in Java; this SO user spends more time learning Java instead of answering C++ questions.

User profiles on SOC provide detailed information regarding work experience as well as information on the technology associated with each job, in the form of tags ${ }^{26}$ To test whether our estimates are driven by skills mismatch, we focus on users who switch to new jobs with similar sets of technologies based on the tags. First, we define a measure of skill-similarity between jobs ${ }^{27}$ Then we re-estimate the DD regressions separately based on the skill-similarity measures. We find that those who switch to new jobs with similar technology also experience a significant drop of Answers over Edits activity. For robustness checks, alternative measures of job similarity are used and all give similar results 28

We also compare the job titles of the old and new jobs. New jobs with the same job title as the old ones are associated with similar responsibilities and availability. These job changes are least likely to be affected by the integer-constraint problem. The DD estimates using this sample are consistent to the baseline results using the full sample. That is to say, the finding is consistent to the career-concerns hypothesis.

\subsection{Dynamic Selection Effects (Ashenfelter's Dip)}

The variations in Figure 4 can also be explained by dynamic selection effects, which says that the sample of job switchers are selected due to a special event prior to the job change that only affects the treatment group but not the control group. This hypothesis is commonly referred to in labor economics literature as Ashenfelter's Dip (AD) 29

Suppose that contributors experience random shocks in the number of Answers and Edits in each period, and suppose that a higher number of Answers can significantly improve the chance of getting job offers (i.e. $p^{\prime}\left(r_{t}\right)$ is large enough). Then the sample of job shifters tends to include those who experience a large Answers shock in periods immediately preceding a job change. In that case, the "bump" in the number of Answers before job changes (as the one in Figure 4) is purely caused by the selection into treatment from

\footnotetext{
${ }^{26}$ Users create tags by attaching relevant terminology to each job to convey technological experience. In our sample, the average number of tags for each job is 5.32. Tags are typically organized into three types: programming languages (e.g. Python, Java), packages/libraries/routines (e.g. NumPy, Matplotlib), functionality (e.g. Data Analysis, Plotting).

${ }^{27}$ Let the set of tags associated with the new job be $S 1$, and those with the old job be $S 0$. We define JobSimilarity $\equiv$ Size $(S 0 \cap S 1)$ $\overline{(\operatorname{Size}(S 0)+\operatorname{Size}(\mathrm{S} 1)) / 2}$.

${ }^{28}$ Detailed regression results can be found in the Online Appendix.

${ }^{29}$ In a more general econometric setting, AD can be considered as a source of endogeneity through reverse causality or selection into treatment. Please refer to Ashenfelter (1978) for a detailed discussion.
} 

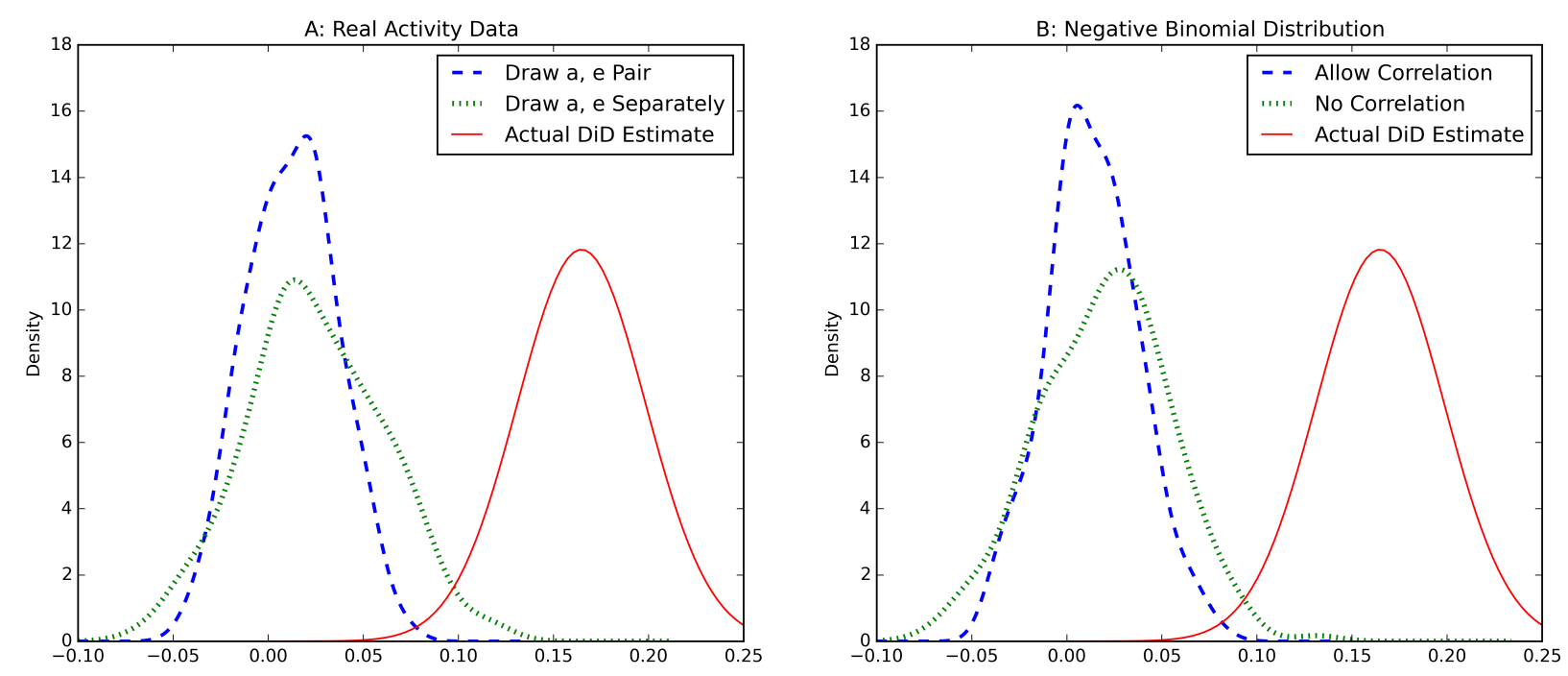

FIGURE 9: Density Plot of Simulated Difference-In-Differences Estimates

random activities, not by changes in user behavior in response to incentives.

This alternative hypothesis also touches on the issue of reverse causality or selection into treatment in which $A_{t-1}$ causes NewJob $_{t}$. In the classical AD problem in Ashenfelter (1978), the dip is assumed to be due to random shocks. Therefore, the problem can be solved by using periods further away from the time of treatment or by matching treatment group with a properly selected control group who also experience a similar shock. However, in our analysis, the "bump" (or the "reversed" dip) in Figure 4, is central to the career-concerns hypothesis. In essence, we are estimating the size of the "bump" and interpret it as a behavioral response by contributors due to career concerns, rather than a design problem with selection into treatment from random shocks.

Identification of $A D$ vs. Career Concerns. We argue, by means of numerical simulations, that AD does not provide compelling evidence against the career-concerns story. Would the estimates be large enough to reject the career concerns hypothesis? If not, under what conditions would we be able to do so?

First, we draw random Answers and Edits activities following a certain distribution (as well as bootstrapping from the actual activities) and then simulate job change status given a likelihood function of job changes. The simulation is then repeated $R$ times and the DD estimates are calculated and plotted. The comparison between simulated estimates and the actual DD estimate can help us to examine whether careerconcerns hypothesis can be rejected in favor of $\mathrm{AD}$ hypothesis ${ }^{30}$ With each simulation, we conduct the DD analysis and plot all the estimates using kernel density plot.

The simulation results are plotted in Figure 9. Panel A plots simulated DD estimates using bootstrapped

\footnotetext{
${ }^{30}$ Detailed simulation method can be found in the Online Appendix.
} 
Answers and Edits (i.e. drawn directly from the actual activity), both in pairs (blue line) and separately (green line). The red line plots the distribution of the actual DD estimate from column 1 of Table 2, with a mean of 0.1627 and a standard deviation of 0.033 . In Panel B, instead of drawing random activity directly from the actual activity, we first fit two negative binomial distributions for Answers and Edits activities. Neither simulated estimates are significantly away from zero. i.e. the DD estimate of .1627 cannot be explained by selection into a job change due to random activities.

Simulations using Answers and Edits drawn separately give a wider range of DD estimates than those using data drawn in pairs. In reality, the number of Answers and Edits given in a month by a contributor is always correlated since both correlate with the time spent on $\mathrm{SO}^{31}$ If the two activities are perfectly correlated, then simulated DD always gives zero estimates. However, Answers and Edits are uncorrelated when drawn independently, thus it's more likely to observe high levels of Answers activity with low Edits activity.

Another reason $\mathrm{AD}$ problem doesn't invalidate the career-concerns hypothesis is the small effect of Answers activity on new job offers. Though unable to accurately estimate this effect due to the presence of endogenous variables, we obtain an upper bound of the true value. The fact that we cannot reject the careerconcerns hypothesis using the upper bound estimate gives us even more confidence in our conclusion.

We also build a simple logit model where Answers activity leads to job offers. We show that in order to mimic the estimates of our main result, the effect of Answers activity on job offers would be unreasonably large 32

\subsection{External Validity}

External validity is one of the most common challenges for empirical studies without a randomly selected sample. Our research is no exception. In fact, our sample is not representative of all SO contributors in terms of total online activities. Stack Overflow, like any platforms that rely on user-generated content, shows a long-tail pattern that the majority of users contribute very little content, and the contributors in our sample are drawn from a much more active population. Without the less active contributors in our analysis, we believe that our results still provide valuable information to platform managers. First, on SO, 10\% of the users contribute roughly $90 \%$ of all contents. These active contributors are the core users that SO cares about most. Our result unravels one of the motivations that drives user activity. Second, we believe that platforms can potentially motivate less active users through career concerns, due to the existing information

\footnotetext{
${ }^{31}$ The actual correlation between Answers and Edits is 0.564.

${ }^{32}$ Details can be found in the Online Appendix.
} 
TABLE 6: EFFects Of CAReER Concerns - DATA From Linkedin Profiles

\begin{tabular}{lcccc}
\hline \hline & $(1)$ & $(2)$ & $(3)$ & $(4)$ \\
& $y=a$ & $y \in\{a, e\}$ & $y=v$ & $y \in\{v, e\}$ \\
\hline NewJob $(S)$ & $-0.165^{* * *}$ & -0.040 & $-0.185^{* * *}$ & -0.040 \\
& $(0.06)$ & $(0.04)$ & $(0.07)$ & $(0.04)$ \\
NewJob $(S) \times$ Answer $(A)$ & & $-0.125^{*}$ & & $-0.146^{*}$ \\
& & $(0.07)$ & & $(0.08)$ \\
\hline Contributors & 197 & 197 & 197 & 197 \\
$N$ & 1396 & 2792 & 1396 & 2792 \\
$R^{2}$ & 0.012 & 0.008 & 0.011 & 0.008 \\
\hline \hline
\end{tabular}

asymmetry problem between job seekers and employers.

Another challenge to the external validity touches on the central element that allows us to conduct this research: the links between $\mathrm{CV}$ and the online activities. Without this link, online activities and real-life job switching events cannot be connected because contributors often adopt pseudonyms. The link is found through SOC profiles. SOC first sends out invitations to active contributors on SO. Then an invited user chooses whether to accept the invitation as well as provide a link to her SO page on the SOC profile. Therefore, a primary concern is that those who accept the invitation and provide a link between their SO and SOC profiles are those who are most interested in ability-signaling through SO activities. In other words, our result that SO contributors respond to career concerns might not apply to other active users who chose not to open an SOC profile or not to provide a link between their SO and SOC profiles.

To address this concern, we propose a way to test the external validity: we searched for indirect links between user CVs and user SO histories. Specifically, we click through on an SO profile until we find a link to the user's LinkedIn page. Once there, we analyze the user's work history from the LinkedIn profile to determine the time of job change.

We explicitly exclude from this sample all users with an SOC profile and those who provide a direct link to LinkedIn. We do so in order to address the concern that a link to SO may result from an endogenous user choice and bias the sample. This alternative sample may be biased against users whose contributions to SO are dependent on career concerns.

Using the same regression specification as in Section 6, Table 6 shows the result of the DD analysis with data collected using the indirect-link approach. Column 2 shows a DD estimate of $12.5 \%$, which is slightly smaller than the estimates in our baseline result in Table 2. The estimates are also less statistically significant due to a smaller sample of 197 contributors.

Admittedly, the test of external validity using the indirect-link approach is not without limitations. Many 
job application processes are conducted through emails or through internal application systems. Moreover, for many SO contributors the links between CV and SO profiles do not exist (or could not be found by us). Nevertheless, our additional results provide additional evidence of career concerns that is consistent with our baseline results.

\section{Discussion and Concluding Remarks}

In this paper, we analyze data from the online Q\&A site Stack Overflow (SO) and show that career concerns provide a strong incentive for users to contribute, namely to answer questions posted on the various SO boards. Our strategy for identifying career-concern-based incentives is to estimate the effect of a job change. Our regressions estimates suggest that achieving the goal of switching to a new job leads users to decrease their contribution to SO; and that a drop of about $12.5-16.5 \%$ can be assigned to a drop in career concerns. This value is both statistically and economically significant. We discuss and test the validity of the identifying assumption by showing evidence related to our career-concerns hypothesis as well as alternative explanations.

While our results pertain to a particular reputation mechanism in a particular online platform, we believe our results have wider application. First, a central issue in organizational behavior is the relative importance of intrinsic and extrinsic motivation. Our paper contributes to this debate by showing the importance of a particular aspect of extrinsic motivation: career concerns. Although we are unable to put an exact number on the relative importance of the different types of motivation, our estimates show that the magnitude of extrinsic motives for contributions to Stack Overflow is considerable.

Second, we contribute to the analysis of Q\&A platforms. Many successful corporations rely on such platforms as a source of value. For example, Amazon reviews provide value to Amazon shoppers; Apple's technical help Q\&A boards add value to Apple products; and Yelp's restaurant reviews provide value to restaurant goers. In this context, an important question is how to provide incentives for extensive and highquality user contributions. Our paper contributes to this analysis by showing how a reputation system can greatly contribute to the success of a Q\&A platform.

More generally, our paper contributes to the understanding of the increasingly important phenomenon of crowdsourcing. Crowdsourcing is broadly understood as the acquisition, at low or zero economic cost, of information or services from customers, users, or other unspecified third parties at low or zero economic cost. Media sites such as CNN or the New York Times make frequent and increasing use of this type of source. As competing content aggregators fight for this rich supply of original information and content, 
the design of incentive mechanisms such as Stack Overflow's reputation system may provide an important competitive edge.

A specific managerial implication of our results pertains to online education platforms such as HBX. One problem these platforms need to solve is scalability: for example, how to answer student questions when the number of students increases by one or two orders of magnitude with respect to typical brickand-mortar class sizes. One possible solution is to resort to AI systems (witness, for example, the 2016 Watson-based experiment in Georgia Tech's Computer Science class). An alternative solution is to rely more heavily on crowdsourcing from users themselves (e.g., have students answer each other's questions, or grade each other's quizzes).

In this context, reputation mechanisms increase user motivation to provide many and good answers (as in Stack Overflow). Moreover, the information contained in the reputation scores themselves adds value to the platform, to the extent that it is used as a job-matching mechanism (as in Stack Overflow Careers). One of the purposes of online education platforms is to certify the graduates' ability. From the point of view of an employer, having access to the information contained in the platform's reputation mechanism greatly enhances the knowledge of the job candidate's abilities. In this sense, the value created by a reputation mechanism is two-fold: it increases the quality of services provided by the platform to students; and it increases the quality of services provided to employers ${ }^{33}$

Finally, as mentioned earlier, the issue of career concerns is central to the discussion of the viability of open-source software. Understanding motivation, as Lerner and Tirole (2002) aptly put it, will "provide lenses through which the structure of open source projects, the role of contributors, and the movement's ongoing evolution can be viewed." Is there any hope for quality code to be written by someone who is not being paid? To the extent that peer recognition may lead to employer recognition, the answer may be yes. Although our paper does not deal with open source software directly, our results suggest that thoughtfullydesigned reputation mechanisms may enhance the link between effort and employer recognition in ways that informal peer recognition may not achieve.

\footnotetext{
${ }^{33}$ An additional managerial implication is that it may be optimal to introduce some form of depreciation in reputation mechanisms such as StackOverflow's: as Holmostrom (1982) argues, an agent's incentive to signal quality decreases as their audience becomes better informed about the agent's ability. Introducing a depreciation rate into an agent's reputation score may help keeping the agent "on his or her toes". The downside is that the discounted value of reputation increases at time $t$ may be lower in the presence of a reputation system with depreciation. While this falls outside of the present paper's scope, we believe this is an interesting research question.
} 


\section{References}

Algan, Y, Y Benkler, and M F Morell. 2013. "Cooperation in a Peer Production Economy Experimental Evidence from Wikipedia.” Working Paper, Department of Economics, Sciences Po .

Ariely, Dan, Anat Bracha, and Stephan Meier. 2009. "Doing Good or Doing Well? Image Motivation and Monetary Incentives in Behaving Prosocially.” American Economic Review 99 (1):544-555.

Ashenfelter, Orley. 1978. "Estimating the Effect of Training Programs on Earnings." The Review of Economics and Statistics 60 (1):47-57.

Ashraf, N, O Bandiera, and Scott S Lee. 2014. "Do-Gooders and Go-Getters: Career Incentives, Selection, and Performance in Public Service Delivery." .

Athey, S and G. Ellison. 2014. "Dynamics of Open Source Movements." Journal of Economics \& Management .

Benabou, Roland and Jean Tirole. 2003. "Intrinsic and extrinsic motivation.” 70 (3):489-520.

2006. “Incentives and prosocial behavior.” The American Economic Review 96 (5):1652-1678.

Bitzer, Jürgen and Ingo Geishecker. 2010. "Who contributes voluntarily to OSS? An investigation among German IT employees." Research Policy 39 (1):165-172.

Blatter, Marc and Andras Niedermayer. 2008. "Informational hold-up, disclosure policy, and career concerns on the example of open source software development." Disclosure Policy, and Career Concerns on the Example of Open Source Software Development (September 1, 2008) .NET Institute Working Paper (0806).

Chevalier, Judith and Glenn Ellison. 1999. "Career concerns of mutual fund managers." Quarterly Journal of Economics 114 (2):389-432.

Gibbons, Robert and Kevin J Murphy. 1992. "Optimal Incentive Contracts in the Presence of Career Concerns: Theory and Evidence." Journal of Political Economy 100 (3):468-505.

Goes, Paulo B, Chenhui Guo, and Mingfeng Lin. 2016. "Do Incentive Hierarchies Induce User Effort? Evidence from an Online Knowledge Exchange.” Information Systems Research 27 (3):497-516. 
Hann, Il-Horn, Jeffrey A Roberts, and Sandra A Slaughter. 2013. "All Are Not Equal: An Examination of the Economic Returns to Different Forms of Participation in Open Source Software Communities." Information Systems Research 24 (3):520-538.

Holmström, Bengt. 1999. "Managerial Incentive Problems: A Dynamic Perspective." Review of Economic Studies 66 (1):169-182.

Huang, Peng and Zhongju Zhang. 2016. "Participation in Open Knowledge Communities and Job-Hopping: Evidence from Enterprise Software.” MIS Quarterly 40 (3):785-806.

Lakhani, Karim R and Eric von Hippel. 2003. "How open source software works: "free" user-to-user assistance." Research Policy 32 (6):923-943.

Lerner, Josh and Jean Tirole. 2002. "Some simple economics of open source." The Journal of Industrial Economics 50 (2):197-234.

— 2005. "The Economics of Technology Sharing: Open Source and Beyond.” The Journal of Economic Perspectives 19 (2):99-120.

Luca, Michael and Georgios Zervas. 2015. "Fake It Till You Make It: Reputation, Competition, and Yelp Review Fraud.” Working Paper, Harvard Business School .

Miklós-Thal, J and H Ullrich. 2015. "Career Prospects and Effort Incentives: Evidence from Professional Soccer.” Management Science .

Osterloh, Margit and Sandra Rota. 2007. "Open source software development—Just another case of collective invention?" Research Policy 36 (2):157-171.

Roberts, Jeffrey A, Il-Horn Hann, and Sandra A Slaughter. 2006. "Understanding the Motivations, Participation, and Performance of Open Source Software Developers: A Longitudinal Study of the Apache Projects." Management Science 52 (7):984-999.

Spiegel, Yossi. 2009. "The incentive to participate in open source projects: a signaling approach.” Working Paper, NET Institute .

Titmuss, Richard Morris and others. 1970. "The gift relationship. From human blood to social policy.” The gift relationship. From human blood to social policy. . 
von Krogh, Georg, Stefan Haefliger, Sebastian Spaeth, and Martin W Wallin. 2012. "Carrots and Rainbows: Motivation and Social Practice in Open Source Software Development.” MIS Quarterly 36 (2).

von Krogh, Georg and Eric von Hippel. 2006. "The Promise of Research on Open Source Software." Management Science 52 (7):975-983.

Zhang, Xiaoquan and Feng Zhu. 2011. "Group size and incentives to contribute: A natural experiment at Chinese Wikipedia." American Economic Review 101 (4):1601-1615. 


\section{Online Appendix}

\section{Proof of Proposition 1}

Proof of Proposition 1 The value function for the two states $(s=0,1)$ can be written as the following:

$$
\begin{aligned}
& V_{t}(0)=\max _{w_{t}, a_{t}, e_{t}} g_{0}\left(w_{t}\right)+f\left(a_{t}, e_{t}\right)+\delta p\left(r_{t}\right) V_{t+1}(1)+\delta\left(1-p\left(r_{t}\right)\right) V_{t+1}(0) \\
& V_{t}(1)=\max _{w_{t}, a_{t}, e_{t}} g_{1}\left(w_{t}\right)+f\left(a_{t}, e_{t}\right)+\delta V_{t+1}(1)
\end{aligned}
$$

This model has infinite time periods and it represents one job switching process (i.e. $s=0$ to $s=1$ ). So the value functions above can be simplified by removing the time subscript and focusing on the two states as following:

$$
\begin{aligned}
& V_{0}=\max _{w_{0}, a_{0}, e_{0}} g_{0}\left(w_{0}\right)+f\left(a_{0}, e_{0}\right)+\delta p\left(r_{t}\right) V_{1}+\delta\left(1-p\left(r_{t}\right)\right) V_{0} \\
& V_{1}=\max _{w_{1}, a_{1}, e_{1}} g_{1}\left(w_{1}\right)+f\left(a_{1}, e_{1}\right)+\delta V_{1}
\end{aligned}
$$

First, we can show that $V_{1}>V_{0}$. Let $x_{s}^{*}$ be the optimal value of control variable $x(x=w, e, a)$ in state $s$ $(s=0,1)$. Suppose that $V_{1} \leq V_{0}$. Then the value functions 8 and 9 can be written as:

$$
\begin{aligned}
& V_{0} \leq \frac{1}{1-\delta}\left[g_{0}\left(w_{0}^{*}\right)+f\left(a_{0}^{*}, e_{0}^{*}\right)\right] \\
& V_{1}=\frac{1}{1-\delta}\left[g_{1}\left(w_{1}^{*}\right)+f\left(a_{1}^{*}, e_{1}^{*}\right)\right]
\end{aligned}
$$

When $s=1$, the optimal time allocation $x_{0}^{*}$ is still feasible. So by choosing $x_{1}=x_{0}^{*}$ when $s=1$ results a strictly higher value of $V_{1}$, which contradicts with $V_{1} \leq V_{0}$. Thus it must be

$$
V_{1}>V_{0}
$$

The homotheticity of $f(a, e)$ means that it can be transformed as $e f(k, 1)$ where $k=\frac{a}{e}$. The second argument in $f(k, 1)$ will be kept as a constant one, which means that we can simplify the notation even further as $e f(k)$. The value functions become: 


$$
\begin{aligned}
& V_{0}=\max _{w_{0}, a_{0}, e_{0}} g_{0}\left(w_{0}\right)+e_{0} f\left(k_{0}\right)+\delta p\left(r_{t}\right) V_{1}+\delta\left(1-p\left(r_{t}\right)\right) V_{0} \\
& V_{1}=\max _{w_{1}, a_{1}, e_{1}} g_{1}\left(w_{1}\right)+e_{1} f\left(k_{1}\right)+\delta V_{1}
\end{aligned}
$$

At state $s$, the agent maximizes $V_{s}$ subject to $w+e+a=T$. So the first-order conditions of the Lagrangian with respect to $w, a$, and $e$ at $s=1$ are given by:

$$
\begin{aligned}
& \lambda_{1}=g_{1}^{\prime}\left(w_{1}\right) \\
& \lambda_{1}=f^{\prime}\left(k_{1}\right) \\
& \lambda_{1}=f\left(k_{1}\right)-k_{1} f^{\prime}\left(k_{1}\right)
\end{aligned}
$$

where $\lambda_{1}$ is the Lagrange multiplier in state 1 . At $s=0$, we have

$$
\begin{aligned}
& \lambda_{0}=g_{0}^{\prime}\left(w_{0}\right) \\
& \lambda_{0}=f^{\prime}\left(k_{0}\right)+\delta\left(V_{1}-V_{0}\right) p^{\prime}\left(r_{t}\right) \\
& \lambda_{0}=f\left(k_{0}\right)-k_{0} f^{\prime}\left(k_{0}\right)
\end{aligned}
$$

Equations 14 and 15 imply that $\left(1+k_{1}\right) f^{\prime}\left(k_{1}\right)-f\left(k_{1}\right)=0$, and equations 17 and 18 imply that $(1+$ $\left.k_{0}\right) f^{\prime}\left(k_{0}\right)-f\left(k_{0}\right)=-\delta\left(V_{1}-V_{0}\right) p^{\prime}\left(r_{t}\right)$, which is less than 0 if and only if $p^{\prime}\left(r_{t}\right)>0$. Then based on these two results, we have the following inequality:

$$
\left(1+k_{1}\right) f^{\prime}\left(k_{1}\right)-f\left(k_{1}\right)>\left(1+k_{0}\right) f^{\prime}\left(k_{0}\right)-f\left(k_{0}\right)
$$

Since $f^{\prime}>0$ and $f^{\prime \prime}<0$, it's straight-forward to show that $h(x)=(1+x) f^{\prime}(x)-f(x)$ decreases in $x$. Thus we have

$$
k_{1}<k_{0} \quad \text { or } \quad \frac{a_{1}}{e_{1}}<\frac{a_{0}}{e_{0}}
$$

Regarding the first part of the Proposition, it helps to first compare equations 15 and 18 . The convexity property of $f$ function imply that $f(x)-x f^{\prime}(x)$ increases in $x$. Together with the second part of the 
proposition $k_{1}<k_{0}$, we get $\lambda_{1}<\lambda_{0}$. With equations 13 and 16 , we get the following inequality:

$$
g_{1}^{\prime}\left(w_{1}\right)<g_{0}^{\prime}\left(w_{1}\right)
$$

$g_{1}^{\prime}>g_{0}^{\prime}$ implies that $g_{1}^{\prime}\left(w_{1}\right)>g_{0}^{\prime}\left(w_{1}\right)$. Together with 20 we get $g_{0}^{\prime}\left(w_{1}\right)<g_{1}^{\prime}\left(w_{1}\right)<g_{0}^{\prime}\left(w_{0}\right)$, which means that $w_{1}>w_{0}$. Since $a_{0}+e_{0}=T-w_{0}$ and $a_{1}+e_{1}=T-w_{1}$ and $\frac{a_{1}}{e_{1}}<\frac{a_{0}}{e_{0}}$, we get $a_{1}<a_{0}$.

Proof of Proposition 2 From the proof of Proposition 1, we have $h(x)=(1+x) f^{\prime}(x)-f(x)$, which is a function decreasing in $x$. We also have the following equation linking $k_{0}$ and $r_{t}$ :

$$
h\left(k_{0}\right)=\left(1+k_{0}\right) f^{\prime}\left(k_{0}\right)-f\left(k_{0}\right)=-\delta\left(V_{1}-V_{0}\right) p^{\prime}\left(r_{t}\right)
$$

For any $0<r_{t-1}<r_{t-1}^{\prime}$,

$$
\begin{aligned}
& \left.h\left(k_{0}\right)\right|_{r_{t-1}}=-\delta\left(V_{1}-V_{0}\right) p^{\prime}\left(r_{t-1}+a_{t}\right) \\
& \left.h\left(k_{0}\right)\right|_{r_{t-1}^{\prime}}=-\delta\left(V_{1}-V_{0}\right) p^{\prime}\left(r_{t-1}^{\prime}+a_{t}\right)
\end{aligned}
$$

Since $p^{\prime}\left(r_{t}\right)>0$ and $p^{\prime \prime}\left(r_{t}\right)<0$, it follows that

$$
\left.h\left(k_{0}\right)\right|_{r_{t-1}}<\left.h\left(k_{0}\right)\right|_{r_{t-1}^{\prime}}
$$

That is to say, $h\left(k_{0}\right)$ increases in $r_{t-1}$. Together with the fact that $h\left(k_{0}\right)$ decreases in $k_{0}$, we get $k_{0}$ decreases in $r_{t-1}$, namely,

$$
\left.\frac{a_{t}}{e_{t}}\right|_{s=0, r_{t-1}}>\left.\frac{a_{t}}{e_{t}}\right|_{s=0, r_{t-1}^{\prime}}
$$

The proof of the second part of Proposition 2 is omitted since it closely resembles that of the first part.

\section{Data Selection}

We focus on a set of users that satisfy a series of criteria required by our empirical test: 
TABLE 7: MONTH-TO-MONTH COMPARISON: FIRST DiFFERENCES

\begin{tabular}{|c|c|c|c|c|c|c|}
\hline \multirow[b]{2}{*}{ Period -20 } & \multicolumn{2}{|c|}{$\begin{array}{c}(1) \\
y=\text { Answers }\end{array}$} & \multicolumn{2}{|c|}{$\begin{array}{c}(2) \\
y=\text { Edits }\end{array}$} & \multicolumn{2}{|c|}{$\begin{array}{c}(3) \\
y=\text { Votes }\end{array}$} \\
\hline & $-0.222 * * *$ & $(0.03)$ & $-0.142 * * *$ & $(0.02)$ & $-0.229 * * *$ & $(0.03)$ \\
\hline Period -19 & $-0.237 * * *$ & $(0.03)$ & $-0.162 * * *$ & $(0.02)$ & $-0.264 * * *$ & $(0.03)$ \\
\hline Period -18 & $-0.234 * * *$ & $(0.03)$ & $-0.149 * * *$ & $(0.02)$ & $-0.269 * * *$ & $(0.03)$ \\
\hline Period -17 & $-0.190^{* * *}$ & $(0.03)$ & $-0.158 * * *$ & $(0.02)$ & $-0.223 * * *$ & (0.03) \\
\hline Period -16 & $-0.204 * * *$ & $(0.03)$ & $-0.134 * * *$ & $(0.02)$ & $-0.223 * * *$ & $(0.03)$ \\
\hline Period -15 & $-0.186 * * *$ & $(0.03)$ & $-0.120 * * *$ & $(0.02)$ & $-0.210 * * *$ & $(0.03)$ \\
\hline Period -14 & $-0.170^{* * *}$ & $(0.03)$ & $-0.105^{* * *}$ & $(0.02)$ & $-0.196 * * *$ & (0.03) \\
\hline Period -13 & $-0.183^{* * *}$ & $(0.03)$ & $-0.113 * * *$ & $(0.02)$ & $-0.196 * * *$ & $(0.03)$ \\
\hline Period -12 & $-0.220 * * *$ & $(0.03)$ & $-0.120 * * *$ & $(0.02)$ & $-0.241 * * *$ & $(0.03)$ \\
\hline Period -11 & $-0.209^{* * *}$ & $(0.03)$ & $-0.114 * * *$ & $(0.02)$ & $-0.233 * * *$ & $(0.03)$ \\
\hline Period -10 & $-0.196^{* * *}$ & $(0.03)$ & $-0.140 * * *$ & $(0.02)$ & $-0.220 * * *$ & $(0.03)$ \\
\hline Period -9 & $-0.212^{* * *}$ & $(0.03)$ & $-0.155^{* * *}$ & $(0.02)$ & $-0.224 * * *$ & $(0.03)$ \\
\hline Period -8 & $-0.203^{* * *}$ & $(0.03)$ & $-0.139 * * *$ & $(0.02)$ & $-0.257 * * *$ & $(0.03)$ \\
\hline Period -7 & $-0.175^{* * *}$ & $(0.03)$ & $-0.111 * * *$ & $(0.02)$ & $-0.179 * * *$ & $(0.03)$ \\
\hline Period -6 & $-0.182^{* * *}$ & $(0.03)$ & $-0.099 * * *$ & $(0.02)$ & $-0.184 * * *$ & $(0.03)$ \\
\hline Period -5 & $-0.140 * * *$ & $(0.03)$ & $-0.089 * * *$ & $(0.02)$ & $-0.158 * * *$ & $(0.03)$ \\
\hline Period -4 & $-0.105^{* * *}$ & $(0.03)$ & $-0.091 * * *$ & $(0.02)$ & $-0.111 * * *$ & $(0.03)$ \\
\hline Period -3 & -0.011 & $(0.03)$ & 0.000 & $(0.02)$ & -0.023 & (0.03) \\
\hline Period -2 (baseline) & 0 & $(-)$ & 0 & $(-)$ & 0 & $(-)$ \\
\hline Period -1 & -0.020 & $(0.03)$ & -0.001 & $(0.02)$ & -0.013 & $(0.03)$ \\
\hline Period 0 & $-0.154 * * *$ & $(0.03)$ & $-0.086^{* * *}$ & $(0.02)$ & $-0.145^{* * *}$ & $(0.03)$ \\
\hline Period 1 & $-0.308^{* * *}$ & $(0.03)$ & $-0.181 * * *$ & $(0.02)$ & $-0.354 * * *$ & $(0.03)$ \\
\hline Period 2 & $-0.224 * * *$ & $(0.03)$ & $-0.082 * * *$ & $(0.02)$ & $-0.268 * * *$ & $(0.03)$ \\
\hline Period 3 & $-0.198 * * *$ & $(0.03)$ & $-0.070 * * *$ & $(0.02)$ & $-0.227 * * *$ & $(0.03)$ \\
\hline Period 4 & $-0.203^{* * *}$ & $(0.03)$ & $-0.072 * * *$ & $(0.02)$ & $-0.226 * * *$ & $(0.03)$ \\
\hline Period 5 & $-0.265^{* * *}$ & $(0.03)$ & $-0.145^{* * *}$ & $(0.02)$ & $-0.288 * * *$ & $(0.03)$ \\
\hline Period 6 & $-0.292 * * *$ & $(0.03)$ & $-0.129 * * *$ & $(0.02)$ & $-0.340 * * *$ & $(0.03)$ \\
\hline Period 7 & $-0.294 * * *$ & $(0.03)$ & $-0.126^{* * *}$ & $(0.02)$ & $-0.326 * * *$ & $(0.03)$ \\
\hline Period 8 & $-0.330 * * *$ & $(0.03)$ & $-0.153 * * *$ & $(0.02)$ & $-0.382 * * *$ & $(0.03)$ \\
\hline Period 9 & $-0.358 * * *$ & $(0.03)$ & $-0.158 * * *$ & $(0.02)$ & $-0.399 * * *$ & $(0.03)$ \\
\hline Period 10 & $-0.374 * * *$ & $(0.03)$ & $-0.167 * * *$ & $(0.02)$ & $-0.425 * * *$ & $(0.03)$ \\
\hline Period 11 & $-0.404 * * *$ & $(0.03)$ & $-0.197 * * *$ & $(0.02)$ & $-0.441 * * *$ & $(0.03)$ \\
\hline Period 12 & $-0.441 * * *$ & $(0.03)$ & $-0.217 * * *$ & $(0.02)$ & $-0.483 * * *$ & $(0.03)$ \\
\hline Period 13 & $-0.461 * * *$ & $(0.03)$ & $-0.207 * * *$ & $(0.02)$ & $-0.523 * * *$ & $(0.03)$ \\
\hline Period 14 & $-0.463 * * *$ & $(0.03)$ & $-0.232 * * *$ & $(0.02)$ & $-0.522 * * *$ & $(0.03)$ \\
\hline Period 15 & $-0.488^{* * *}$ & $(0.03)$ & $-0.230 * * *$ & $(0.02)$ & $-0.533 * * *$ & $(0.03)$ \\
\hline Period 16 & $-0.475^{* * *}$ & $(0.03)$ & $-0.261 * * *$ & $(0.02)$ & $-0.528 * * *$ & $(0.03)$ \\
\hline Period 17 & $-0.483^{* * *}$ & $(0.03)$ & $-0.233 * * *$ & $(0.02)$ & $-0.537 * * *$ & $(0.03)$ \\
\hline Period 18 & $-0.489 * * *$ & $(0.03)$ & $-0.248 * * *$ & $(0.02)$ & $-0.551 * * *$ & $(0.03)$ \\
\hline Period 19 & $-0.479 * * *$ & $(0.03)$ & $-0.246^{* * *}$ & $(0.02)$ & $-0.532 * * *$ & $(0.03)$ \\
\hline Period 20 & $-0.480 * * *$ & $(0.03)$ & $-0.247 * * *$ & $(0.02)$ & $-0.554 * * *$ & $(0.03)$ \\
\hline Seasonality dummy & $\mathrm{x}$ & & $\mathrm{x}$ & & $\mathrm{x}$ & \\
\hline Duration dummy & $\mathrm{x}$ & & $\mathrm{x}$ & & $\mathrm{x}$ & \\
\hline No. of observations & 4646575 & & 4646575 & & 4646575 & \\
\hline$R^{2}$ & 0.047 & & 0.004 & & 0.038 & \\
\hline
\end{tabular}

Notes: This table summarizes the estimates of $\beta_{\tau}$ in regression 6 by using Answers, Edits, and Votes as the dependent variable. $\beta_{\tau}$ measures the differences in logged activities between period $\tau$ and -2 , while controlling for seasonality and duration effects. Period 1 is the first month when a new job starts. Period -2 is used as the (omitted) baseline period since it has the highest activity level. The estimates show that both Answers and Edits activities rise up gradually until three months before a job change, and then both start to drop. The values of $\beta_{\tau}$ are plotted in Figure 5 Robust standard errors in parentheses, clustered at individual-activity type level. $* * * p<0.01, * * p<0.05, * p<0.1$ 
TABLE 8: MONTH-TO-MONTH COMPARISON: DIFFERENCE-IN-DIFFERENCES

\begin{tabular}{|c|c|c|c|c|}
\hline \multirow[b]{2}{*}{ Period -20} & \multicolumn{2}{|c|}{ (1) } & \multicolumn{2}{|c|}{ (2) } \\
\hline & -0.079 & $(0.05)$ & -0.087 & $(0.06)$ \\
\hline Period -19 & -0.075 & $(0.05)$ & $-0.103 *$ & $(0.06)$ \\
\hline Period -18 & $-0.084 *$ & $(0.05)$ & $-0.120 * *$ & $(0.06)$ \\
\hline Period -17 & -0.032 & $(0.05)$ & -0.065 & $(0.05)$ \\
\hline Period -16 & -0.070 & $(0.05)$ & $-0.089 *$ & $(0.05)$ \\
\hline Period -15 & -0.066 & $(0.05)$ & $-0.091 *$ & $(0.05)$ \\
\hline Period -14 & -0.065 & $(0.05)$ & $-0.091 *$ & $(0.05)$ \\
\hline Period -13 & -0.070 & $(0.05)$ & -0.083 & $(0.05)$ \\
\hline Period -12 & $-0.100 * *$ & $(0.05)$ & $-0.121 * *$ & $(0.05)$ \\
\hline Period -11 & $-0.095 * *$ & $(0.05)$ & $-0.119 * *$ & $(0.05)$ \\
\hline Period -10 & -0.055 & $(0.04)$ & -0.080 & $(0.05)$ \\
\hline Period -9 & -0.057 & $(0.04)$ & -0.069 & $(0.05)$ \\
\hline Period -8 & -0.064 & $(0.04)$ & $-0.118 * *$ & $(0.05)$ \\
\hline Period -7 & -0.063 & $(0.04)$ & -0.068 & $(0.05)$ \\
\hline Period -6 & $-0.083^{* *}$ & $(0.04)$ & $-0.085^{*}$ & $(0.04)$ \\
\hline Period -5 & -0.050 & $(0.04)$ & -0.069 & $(0.05)$ \\
\hline Period -4 & -0.015 & $(0.04)$ & -0.020 & $(0.04)$ \\
\hline Period -3 & -0.011 & $(0.03)$ & -0.023 & $(0.04)$ \\
\hline Period -2 (baseline) & 0 & $(-)$ & 0 & $(-)$ \\
\hline Period -1 & -0.020 & $(0.03)$ & -0.012 & $(0.04)$ \\
\hline Period 0 & $-0.068^{*}$ & $(0.04)$ & -0.059 & $(0.04)$ \\
\hline Period 1 & $-0.127 * * *$ & $(0.04)$ & $-0.173 * * *$ & $(0.05)$ \\
\hline Period 2 & $-0.142 * * *$ & $(0.04)$ & $-0.186 * * *$ & $(0.05)$ \\
\hline Period 3 & $-0.128 * * *$ & $(0.04)$ & $-0.157 * * *$ & $(0.05)$ \\
\hline Period 4 & $-0.131 * * *$ & $(0.04)$ & $-0.154 * * *$ & $(0.05)$ \\
\hline Period 5 & $-0.120^{* * *}$ & $(0.04)$ & $-0.143 * * *$ & $(0.05)$ \\
\hline Period 6 & $-0.163 * * *$ & $(0.04)$ & $-0.210 * * *$ & $(0.05)$ \\
\hline Period 7 & $-0.169 * * *$ & $(0.04)$ & $-0.201 * * *$ & $(0.05)$ \\
\hline Period 8 & $-0.178^{* * *}$ & $(0.04)$ & $-0.229 * * *$ & $(0.05)$ \\
\hline Period 9 & $-0.200 * * *$ & $(0.04)$ & $-0.241 * * *$ & $(0.05)$ \\
\hline Period 10 & $-0.207 * * *$ & $(0.04)$ & $-0.258 * * *$ & $(0.05)$ \\
\hline Period 11 & $-0.208 * * *$ & $(0.04)$ & $-0.245 * * *$ & $(0.05)$ \\
\hline Period 12 & $-0.224 * * *$ & $(0.04)$ & $-0.265 * * *$ & $(0.05)$ \\
\hline Period 13 & $-0.254 * * *$ & $(0.04)$ & $-0.316^{* * *}$ & $(0.05)$ \\
\hline Period 14 & $-0.231 * * *$ & $(0.04)$ & $-0.290 * * *$ & $(0.05)$ \\
\hline Period 15 & $-0.258^{* * *} *$ & $(0.04)$ & $-0.303 * * *$ & $(0.05)$ \\
\hline Period 16 & $-0.214 * * *$ & $(0.04)$ & $-0.267 * * *$ & $(0.05)$ \\
\hline Period 17 & $-0.250 * * *$ & $(0.04)$ & $-0.303 * * *$ & $(0.05)$ \\
\hline Period 18 & $-0.241 * * *$ & $(0.04)$ & $-0.302 * * *$ & $(0.05)$ \\
\hline Period 19 & $-0.233^{* * *} *$ & $(0.04)$ & $-0.286 * * *$ & $(0.05)$ \\
\hline Period 20 & $-0.233 * * *$ & $(0.04)$ & $-0.308 * * *$ & $(0.05)$ \\
\hline Seasonality dummy & $\mathrm{x}$ & & $\mathrm{x}$ & \\
\hline Duration dummy & $\mathrm{x}$ & & $\mathrm{x}$ & \\
\hline No. of observations & 9293150 & & 9293150 & \\
\hline$R^{2}$ & 0.034 & & 0.029 & \\
\hline
\end{tabular}

Notes: This table lists the estimates of $\gamma_{\tau}$ in regression 7 by using Answers, Votes, Questions, together with Edits, as the dependent variables. Edits activity is used as the control group. $\gamma_{\tau}$ captures the differences in changes of vote-generating activities relative to changes in Edits between period $\tau$ and -2 . The demeaned values of $\gamma_{\tau}$ are plotted in Figure 6 Robust standard errors in parentheses, clustered at individual-activity type level. *** $p<0.01, * * p<0.05$, * $p<0.1$ 
- Located in the U.S. and Canada: this ensures a more homogenous sample ${ }^{34}$

- Job switchers: the change in the level of career concerns comes from a job switch; we select users who experienced a job change from November 2008 until November 2014, the month when we stopped collecting data. To focus on job switches, we require the gap between two jobs are less or equal to one month. 35

- Active users: for many users, we do not observe any activity on SO during periods of job change; for more accurate estimation, we focus on active users, defined as having at least one Answer and at least one Edit within the four-month period before or after the month of a job change. (in other words, we exclude inactive SO users) 36

- Multiple job switches: Some users experienced more than one switch. More stable employment is associated with a sharper change in career incentives than temporary or transitional employment. So we exclude such switches if they are less than 8 months apart ${ }^{37}$

- Profiles with links to SO: We limit our dataset to users with links to their SO profiles, because the ability to track users' online activities requires this link.

\section{More on Questions Activity}

Questions is another important activity on SO. Here we investigate how Questions activity changes surrounding the event of a job change. In order to plot the average logged activity while controlling for seasonality and duration effects, we plot the demeaned value of $\beta_{\tau}$ from regression 6 in Figure 10 .

Figure 10 shows the rate of Questions asked around the time of a job shift. Unlike Answers and Edits, we observe little changes in the number of Questions over time. Questions activity experiences a slight drop at the end of the old job and then rises right after the starting month of a new job. One possible explanation of the rise is that, more than a reputation-increasing activity, Questions are used a learning tool; and a shift to a new job creates new learning demands (even for new jobs with the same set of technology), an effect that seems to compensate for the higher opportunity cost of time spent on SO as well as the diminished

\footnotetext{
${ }^{34}$ A large fraction of the jobs posted on SOC are located in the United States and Canada.

${ }^{35}$ Results from changes in employment status, i.e. from unemployed to employed, can also be interesting. However, from the CV data, we are unable to distinguish unemployment from other activities such as vacations.

${ }^{36} \mathrm{We}$ also test the robustness of the result by altering the time periods in this selection.

${ }^{37}$ Other criteria are also tested.
} 


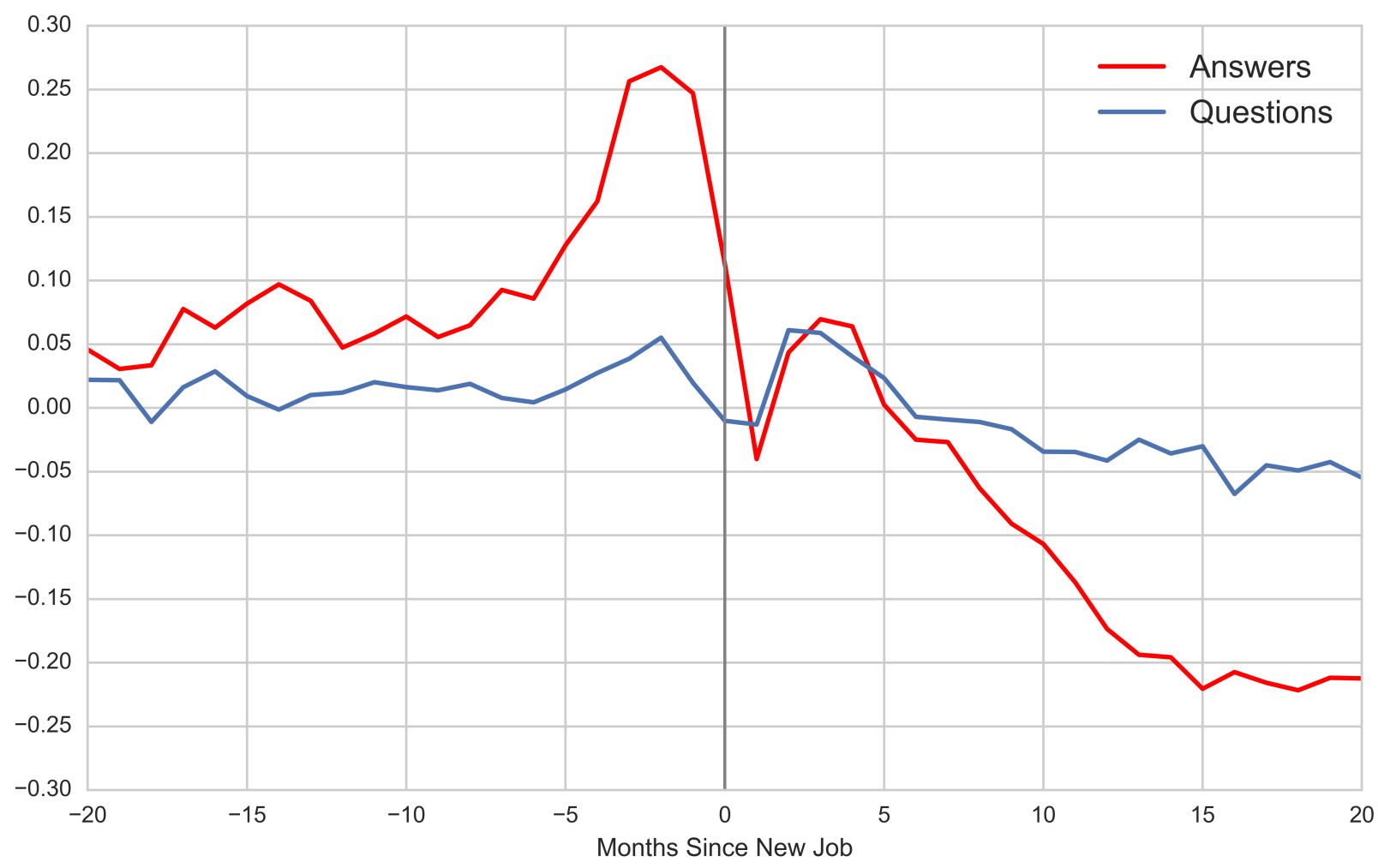

FIGURE 10: Answers and Questions Activity on Stack Overflow

Notes: This figure contrasts how Answers and Questions activity change surrounding the event of a job change. More specifically, it plots the demeaned values of $\beta_{\tau}$ in regression 6 using Answers and Questions as dependent variables, in order to control for seasonality and duration effects.

A contributor can earn reputation points through both Answers and Questions activities through Votes casted by others. One Vote to an Answer rewards 10 points, and one Vote to a Question rewards 5 points. This figure shows that job seekers respond to career incentives through increasing Answers activity, but not through Questions activity. Several explanations can explain this phenomenon: 1. Answers activity is a better way to improve one's reputation online. 2. Employers might consider a job seeker who ask too many Questions as a negative signal. 
incentive to build a reputation. Another possible explanation is that asking Questions might be perceived as inability to solve problems, and thus job seekers avoid asking Questions 38

As mentioned in the main draft, SO users can also receive reputation points through asking Questions, and at the same time, it also takes time to ask a good Question. One might wonder why we focused mostly on Answers rather than Questions activity in our main empirical analysis.

At a conceptual level, the incentive structures behind Questions and Answers/Edits activity are very different. One asks a question in order to get answers (help seeking), and one provides an answer or edit in order to help others (help offering) ${ }^{39}$ When referring to online contribution activities, we meant help-offering, rather than help-seeking, activities; and we test the theory of career concerns to explain the phenomenon that private individuals spend considerable time and effort to help each other.

In the theoretical framework, Questions activity can be considered as part of $w$ (time spent at work), since most Questions are asked to solve real-world problems encountered at work, the schedule of which does not usually change significantly over time. Time availability $(T-w)$ is then allocated to other non-work-related activities (providing Answers and Edits). In other words, Questions are help-seeking tasks essential to one's work that needs to be solved, whereas Answers/Edits are non-essential tasks to one's job and are more subject to a contributor's choices, thus more elastic to changes in time availability. Accordingly, our plots show greater variation in Answers and Edits activity over time but a much smoother trend in Questions activity.

In relation to career concerns, although one can receive votes through Questions activity which can accrue reputation points, we do not think Questions would act as a channel for a job seeker to signal one's quality to potential employers for the following reasons. First, each vote casted to a Question rewards 5 reputation points, which is considerably less than the 10 reputation points rewarded to an Answer and 15 points to a correct Answer. On average, a Question receives 1.83 votes, whereas an Answer receives 2.53 votes. Second, one cannot simply ask random questions: the simple or duplicate questions are often down-voted or deleted which would adversely affect one's online reputation. Third, a typical user profile prominently displays the list of top Answers by default. Although asking good questions is indeed a good quality in certain fields such as academic research, we believe that high-quality Answers provide much more valuable ability signals to employers than other information on the profile 40

\footnotetext{
${ }^{38}$ Please see the Online Appendix for more analysis related to Questions activity.

${ }^{39}$ In relation to the public goods literature, Answers/Edits are more like private contribution to public goods, whereas Questions are more like, although not strictly, private goods, because there would be no Answers without Questions.

${ }^{40}$ In a sense, Answers are like peer-reviewed journal articles, and each up-vote is like a citation.
} 
TABLE 9: EfFects of Job Changes For New Jobs With Similar Technology

\begin{tabular}{|c|c|c|c|c|c|c|}
\hline & \multicolumn{3}{|c|}{ Panel A: $y \in\{$ Answers, Edits $\}$} & \multicolumn{3}{|c|}{ Panel B: $y \in\{$ Votes, Edits $\}$} \\
\hline & $\begin{array}{c}(1) \\
\text { Sim. } \geq 100 \%\end{array}$ & $\begin{array}{c}(2) \\
\operatorname{Sim} . \geq 50 \%\end{array}$ & $\begin{array}{c}(3) \\
\text { Sim. } \geq 0 \%\end{array}$ & $\begin{array}{c}(4) \\
\text { Sim. } \\
\text {. } \\
\end{array}$ & $\begin{array}{c}(5) \\
\text { Sim. } \geq 50 \%\end{array}$ & $\begin{array}{c}(6) \\
\text { Sim. } \geq 0 \%\end{array}$ \\
\hline $\operatorname{NewJob}(S)$ & $\begin{array}{l}-0.013 \\
(0.06)\end{array}$ & $\begin{array}{c}-0.074 * * \\
(0.04)\end{array}$ & $\begin{array}{c}-0.082 * * * \\
(0.02)\end{array}$ & $\begin{array}{l}-0.013 \\
(0.06)\end{array}$ & $\begin{array}{c}-0.074 * * \\
(0.04)\end{array}$ & $\begin{array}{c}-0.082 * * * \\
(0.02)\end{array}$ \\
\hline NewJob $(S) \times$ Answer $/$ Vote $(J)$ & $\begin{array}{c}-0.173^{*} \\
(0.10)\end{array}$ & $\begin{array}{c}-0.122^{*} \\
(0.06)\end{array}$ & $\begin{array}{c}-0.125^{* * *} \\
(0.03)\end{array}$ & $\begin{array}{c}-0.231 * * \\
(0.11)\end{array}$ & $\begin{array}{c}-0.150 * * \\
(0.07)\end{array}$ & $\begin{array}{c}-0.154 * * * \\
(0.04)\end{array}$ \\
\hline Seasonality dummy & $\mathrm{x}$ & $\mathrm{x}$ & $\mathrm{x}$ & $\mathrm{x}$ & $\mathrm{x}$ & $\mathrm{x}$ \\
\hline Duration dummy & $\mathrm{x}$ & $\mathrm{x}$ & $\mathrm{x}$ & $\mathrm{x}$ & $\mathrm{x}$ & $\mathrm{x}$ \\
\hline Contributors & 96582 & 96823 & 97658 & 96582 & 96823 & 97658 \\
\hline No. of observations & 9089694 & 9092762 & 9105016 & 9089694 & 9092762 & 9105016 \\
\hline$R^{2}$ & 0.033 & 0.033 & 0.033 & 0.027 & 0.027 & 0.027 \\
\hline
\end{tabular}

Notes: This table summarizes the DD estimates based on the similarities of technologies used in the old and the new jobs. We introduce a measure of job similarity and conduct separate analysis based on that measure. A job similarity of $100 \%$ means that the new job has exactly the same set of tags as the old one; $0 \%$ means that the old and new jobs have no common tags. The goal is to test whether our DD estimate can be explained by the hypothesis of skills mismatch. The result shows that even for job seekers who switch to new jobs with exactly the same set of technology, the DD estimate is still significant at $-17.3 \%$ (column 1).

Robust standard errors in parentheses, clustered at individual-activity type level. ${ }^{* * *} p<0.01, * * p<0.05, * p<0.1$

\subsection{Skills Mismatch}

Table 9 summarizes the DD estimates using different thresholds of tags similarity. Column 1 focuses 162 job changers whose new jobs have exactly the same tags as the old ones, and DD coefficient gives an estimate of $-20.9 \%$, which is a larger magnitude compared to the estimate of $-16.27 \%$ from our baseline model. Moving to the right, column 1 to 3 and column 4 to 6 gradually lower the thresholds of job similarity and include more job changers in the regression. Column 3 and 6 includes all the users, which is our baseline model.

The results in Table 9 show that job changers who switch to positions with similar skill requirements also experience a similar drop in Answers over Edit activity. The magnitudes of the estimates using various thresholds are also comparable to results from the baseline model. However, user-provided tags information can cause measurement errors to each job. In particular, a new job might require new technologies or programs that are unfamiliar to a job changer, even after controlling for the tags information on the CV.

To further control this effect, we conduct additional analysis using tags related to the actual Questions and Answers on SO. Each question is attached to multiple tags indicating the related languages, libraries or functionality. These tags information gives us more information on whether a programmer is actually asking questions that are previously unfamiliar to her. 
Table 10: Alternative Measure of New Jobs with Similar Technology

\begin{tabular}{|c|c|c|c|c|c|c|}
\hline & \multicolumn{2}{|c|}{ Panel A: 3-Month } & \multicolumn{2}{|c|}{ Panel B: 6-Month } & \multicolumn{2}{|c|}{ Panel C: 12-Month } \\
\hline & $\begin{array}{c}(1) \\
y \in\{a, e\}\end{array}$ & $\begin{array}{c}(2) \\
y \in\{v, e\}\end{array}$ & $\begin{array}{c}(3) \\
y \in\{a, e\}\end{array}$ & $\begin{array}{c}(4) \\
y \in\{v, e\}\end{array}$ & $\begin{array}{c}(5) \\
y \in\{a, e\}\end{array}$ & $\begin{array}{c}(6) \\
y \in\{v, e\}\end{array}$ \\
\hline $\operatorname{NewJob}(S)$ & $\begin{array}{c}-0.433 * * * \\
(0.15)\end{array}$ & $\begin{array}{c}-0.433 * * * \\
(0.15)\end{array}$ & $\begin{array}{c}-0.423 * * \\
(0.18)\end{array}$ & $\begin{array}{c}-0.423 * * \\
(0.18)\end{array}$ & $\begin{array}{c}-0.305^{*} \\
(0.18)\end{array}$ & $\begin{array}{c}-0.305^{*} \\
(0.18)\end{array}$ \\
\hline $\operatorname{NewJob}(S) \times \operatorname{Answer}(A)$ & $\begin{array}{l}-0.247 \\
(0.23)\end{array}$ & $\begin{array}{l}-0.351 \\
(0.26)\end{array}$ & $\begin{array}{l}-0.259 \\
(0.27)\end{array}$ & $\begin{array}{l}-0.297 \\
(0.30)\end{array}$ & $\begin{array}{l}-0.101 \\
(0.30)\end{array}$ & $\begin{array}{l}-0.111 \\
(0.35)\end{array}$ \\
\hline Contributors & 30 & 30 & 26 & 26 & 22 & 22 \\
\hline$N$ & 370 & 370 & 322 & 322 & 286 & 286 \\
\hline$R^{2}$ & 0.134 & 0.134 & 0.120 & 0.108 & 0.059 & 0.047 \\
\hline
\end{tabular}

Notes: This table summarizes the DD estimates based on the similarities of technologies used in the old and the new jobs. We introduce a measure of job similarity and conduct separate analysis based on that measure. A job similarity of $100 \%$ means that the new job has exactly the same set of tags as the old one; $0 \%$ means that the old and new jobs have no common tags. The goal is to test whether our DD estimate can be explained by the hypothesis of skills mismatch. The result shows that even for job seekers who switch to new jobs with exactly the same set of technology, the DD estimate is still significant at $-17.3 \%$ (column 1).

Robust standard errors in parentheses, clustered at individual-activity type level. ${ }^{* * *} p<0.01, * * p<0.05, * p<0.1$

We gather all the tags of the Questions asked by each contributor within M-month $(M=3,6,12)$ after a job change, and compare them to those within 12-month before the job change. Then we conduct DD analysis using contributors who have not asked Questions with any new tags after the job change.

Table 10 includes three panels using tags of Questions from 3, 6, 12-month after job changes as measures of technology. The sample size is quite small since it is very common for a contributor to interact with new tags, both within and across jobs. The DD estimates have the right sign that support our hypothesis of career concerns, although they are all insignificant due to the small sample size.

Admittedly, both approaches (comparing tags listed on CV and tags associated to Questions) are noisy measures of the actual technologies encountered in a new job. A more perfect control of this effect requires much more detailed data on the jobs than those available on a CV. However, we do hope that these two additional robustness checks can help attenuate the concerns related to the inability to contribute due to changes in required skillsets.

\subsection{Switch to a Similar Job (Title)}

The integer-constraint hypothesis argues that a new job might have a work schedule that has small blocks of free time enough for making Edits, but not large blocks of free time for giving Answers. Although without data on the actual work schedule, we can infer work schedule changes based on certain information on a CV. 
TABle 11: Effects Of Job Changes For Switching To Jobs With Same Job Titles

\begin{tabular}{lcccccc}
\hline & Baseline & & \multicolumn{3}{c}{ Same Job Title } \\
\cline { 2 - 3 } \cline { 5 - 7 } & $(1)$ & & $(2)$ & $(3)$ & $(4)$ & $(5)$ \\
\hline NewJob & $-0.074^{* * *}$ & & -0.028 & -0.028 & -0.008 & -0.028 \\
& $(0.02)$ & & $(0.06)$ & $(0.06)$ & $(0.07)$ & $(0.08)$ \\
NewJob $\times$ Answer & $-0.124^{* * *}$ & $-0.181^{*}$ & -0.138 & -0.161 & $-0.244^{*}$ \\
& $(0.03)$ & & $(0.11)$ & $(0.11)$ & $(0.11)$ & $(0.14)$ \\
Seasonality & $\mathrm{x}$ & & $\mathrm{x}$ & $\mathrm{x}$ & $\mathrm{x}$ \\
Duration & $\mathrm{x}$ & & $\mathrm{x}$ & $\mathrm{x}$ & $\mathrm{x}$ \\
\hline Days & - & & - & - & Weekday & Weekend \\
Contributors (DD) & 1301 & & 155 & 155 & 144 & 45 \\
Contributors (ctrl) & 96422 & & - & 96422 & 96422 & 51296 \\
$N$ & 7380646 & & 1992 & 7365094 & 7364828 & 2919924 \\
$R^{2}$ & 0.031 & & 0.010 & 0.031 & 0.031 & 0.043 \\
\hline
\end{tabular}

Notes: Robust standard errors in parentheses, clustered at individual-activity type level. ${ }^{* * *} p<0.01, * * p<0.05,{ }^{*} p<0.1$

In particular, we can focus on jobs that have exactly the same job titles ${ }^{41}$ This type of job changes helps to minimize the influence of changes in the nature of the work performed. The new jobs with the same job title as the old ones are associated with similar responsibilities, thus similar time flexibility, so these job changes are least likely to be affected by integer-constraint problem. If the hypothesis of career concerns is true, then we expect to observe a significant DD estimate using this sample, both from weekday and weekend activities.

Table 11 summarizes the DD estimates. Out of 1301 contributors, 155 (or 12.5\%) of them changes to new jobs that have the exact same job titles as the old ones. Due to the small sample size, most of the results become statistically insignificant but the point estimate tells a story that is consistent career-concerns hypothesis.

Table 11 shows that, for this sample of job changers who are least likely to subject to the integerconstraint problem, the DD estimates are consistent to those with the full sample $(18.1 \%$ without controls and $13.8 \%$ with controls in columns 2 and 3). At the same time, the changes of Edits activity after a job switch are almost negligible (row 1 of columns 2 and 3), indicating a similar time availability before and after the job change. The DD analysis using weekday activity gives a similar result $(16.1 \%$ in column 4); however, the regression using weekend activity gave a large significant estimate of $24.4 \%$ (column 5), implying a large effect of career incentives for this group of contributors.

\footnotetext{
${ }^{41}$ Some of the most common job titles are: Software Engineer, Software Developer, and Web Developer.
} 


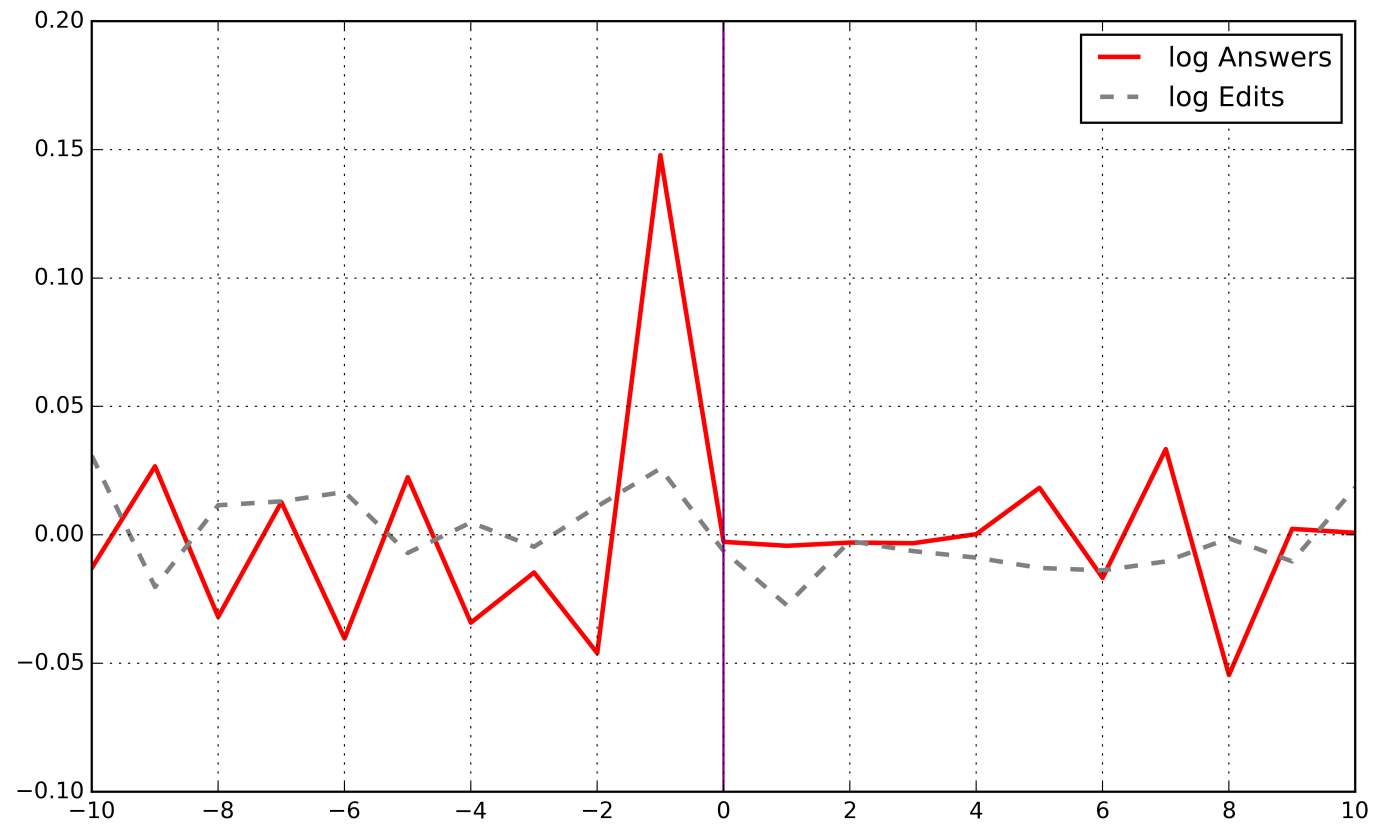

FIGURE 11: Demonstration of Dynamic Selection Effects by Simulation

Notes: This figure demonstrate the potential problem of dynamic selection effects. It is commonly referred to as Ashenfelter's Dip in Labor Economics. Given random shocks of Answers and Edits activity, and given a job changing function that increases in lagged Answers activity with certain parameter value, one can simulate job changes and plot a graph similar to what we observed using actual SO activity data.

\subsection{Dynamic Selection Effects (Ashenfelter's Dip)}

The problem of AD can be shown through simulating job change events using random activities together with a certain likelihood function of job changes. Figure 11 plots the mean logged levels of simulated activities over time. It shows a clear increase in Answers activity before a job change, followed by a reduction afterwards, which resembles Figure 4 . In this case, even though a DD specification can generate a significant estimate, it is clearly not due to career concerns. The increase in Answers activity before the job change is purely due to a selection of periods with large Answers shocks; the reduction after the job change is purely due to a recovery from the shock.

Choices of Simulation Inputs. The simulation requires two main inputs: 1. Random draws of Answers and Edits activities 2. Probability of a job change given Answers activity from the previous period. The simulation parameters can significantly affect the simulation results.

For the first part of the input, we draw Answers and Edits activities from the actual monthly activities ${ }^{42}$ In order to keep the data as clean as possible - i.e., without potential effects of career incentives - we use the activities during a period that is at least five months away from a job change. We draw Answers and

\footnotetext{
${ }^{42}$ This method is also known as bootstrapping, or random sampling with replacement.
} 
Edits both in pairs and separately. The results using these two approaches help us compare the typical DD approach in settings such as AD to the DD approach developed earlier in the paper. As a robustness check, we also conduct simulations drawn from two separate negative binomial distributions which are fitted to the actual activities, for cases both with and without correlation between the two distributions.

For the second part of the input, we use parameters from a logistic regression of job change status on the lagged Answers activity ${ }^{43}$ There is an endogeneity problem, since job search intensity correlates with both job change status and Answers activity. Unfortunately, we are unable to solve it due to lack of data on both job search intensity and job offers received. However, the estimate from a regression with an endogeneity problem should provide us the upper bound of the true value, which is the worst-case scenario to the careerconcerns hypothesis. In other words, by not correcting for endogeneity we are stacking the cards against our preferred hypothesis.

For each simulation method, we simulate the DD results $R=200$ times. Each simulation includes 1500 job switches which mimics our original DD analysis.

Parameter Values Required to Reject Career Concerns. Figure 9 shows a significant gap between the simulated results and the actual DD estimates, which favors the career-concerns hypothesis. However, the simulated results crucially depend on the two inputs discussed above. In this subsection, we adjust the second input, the probability of job changes given a certain level of Answers activity, and calibrate the parameters in order to mimic the actual DD estimate.

The job change probability is modeled using the following simple logit model:

$$
\operatorname{Pr}(J C)=\operatorname{Logit}(\alpha+\beta * A)=\frac{\exp (\alpha+\beta * A)}{1+\exp (\alpha+\beta * A)}
$$

First, we calibrate $\alpha$ while holding $A=0$ by matching the simulated job length to the distribution of actual job lengths from the data. With the calibrated value $\hat{\alpha}=-3.07$, the unconditional rate of job change is $\frac{\exp (\hat{\alpha})}{1+\exp (\hat{\alpha})}=4.43 \%$. Then, holding $\alpha=-3.07$, we simulate DD estimates for different values of $\beta$. For each $\beta$, we run the simulation for $R=100$ times and plot the average values in Figure 12

Figure 12 shows that the value of $\beta=22.76 \%$ produces a simulated DD estimate that is closest to our actual DD estimate of $16.27 \%$. $\beta=22.76 \%$ means that one log unit increase of number of Answers increases the likelihood of a job change by $22.76 \%$. For our sample of 1,031 SOC users, an average use contributes 4.05 Answers per month. Thus $\beta=22.76 \%$ means that if a job seeker contributes 6.88 Answers, then the

\footnotetext{
${ }^{43}$ We also checked simulation results using alternative specifications, e.g. including or excluding reputation level, lagged Edits, etc. They all produce similar results.
} 


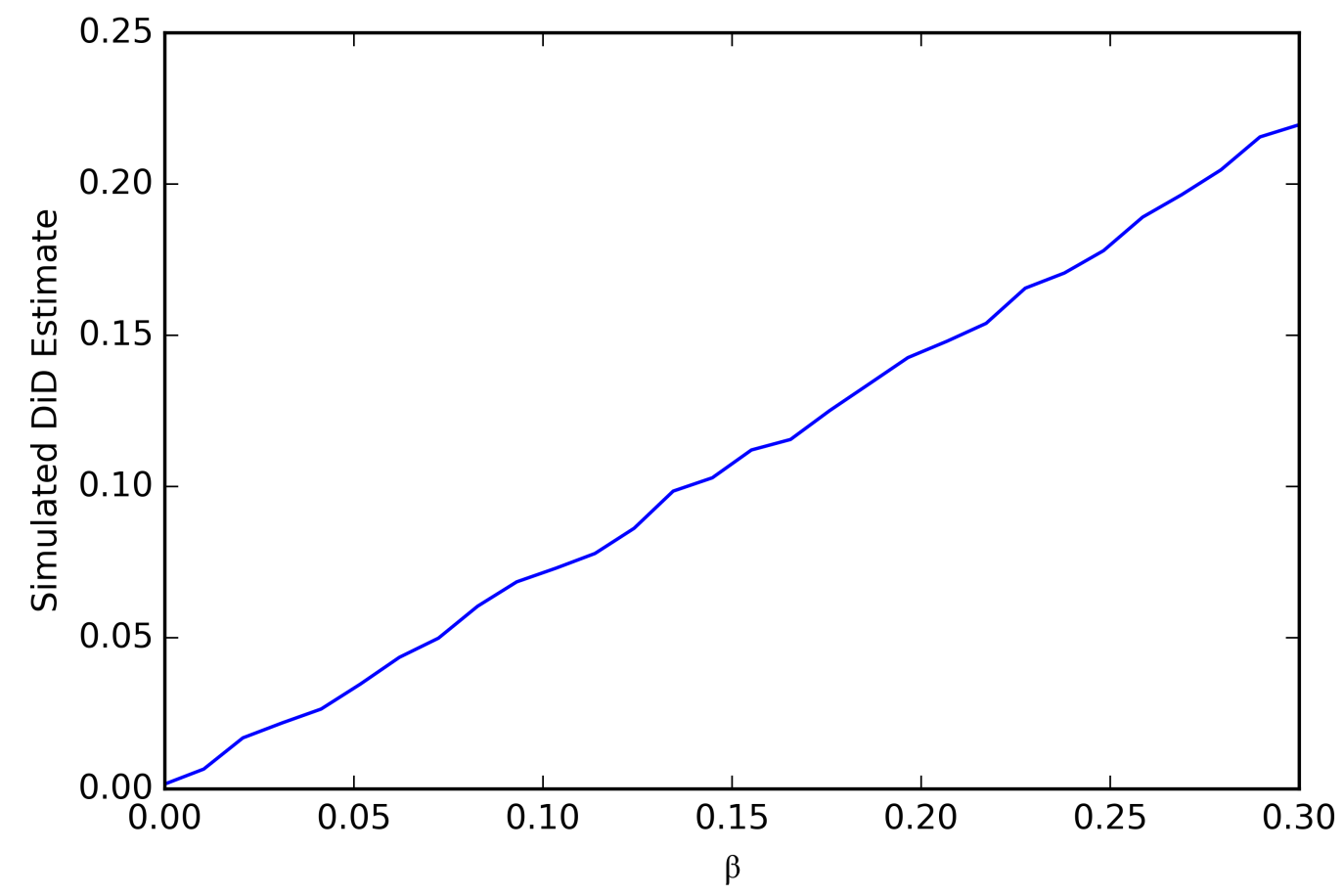

FIGURE 12: Average Simulated DD Estimates Given Values of $\beta$

Notes: The figure plots average simulated DD estimates by using different values of $\beta$. It shows the value of $\beta$ needed in order for dynamic selection effects to produce a simulated DD estimate of $16.27 \%$. $\beta$ is the coefficient of Answers activity in the probability function of encountering a job change event. 
chance of changing to a new job increases by $22.76 \%{ }^{44}$ Given the relatively low cost of providing Answers on SO, the benefit of the additional online activities is enormous, which is not the case in reality simply from observation. Therefore, we conclude that the magnitude of the causal effect, which is represented by $\beta$, is over-estimated. That is to say, in order to reject the career-concerns hypothesis, the parameter value needed in the job change function is too large to be reasonable.

${ }^{44}$ One $\log$ unit increase is roughly an increase of $170 \%$. So one log unit increase for a typical user is approximately $4.05 \times 170 \%=$ 6.88 . 